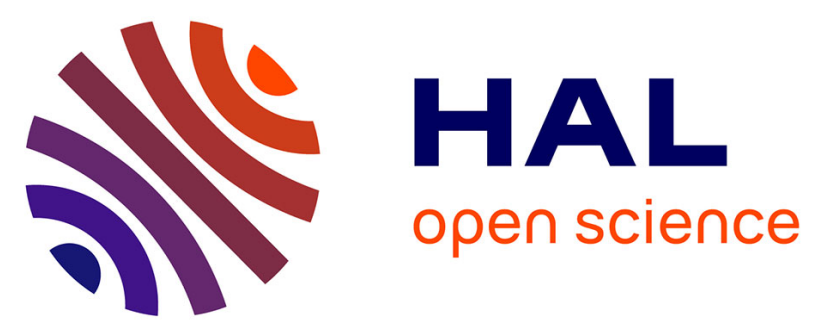

\title{
The root-knot nematode effector MiPDI1 targets a stress-associated protein (SAP) to establish disease in Solanaceae and Arabidopsis
}

Jianlong Zhao, Joffrey Mejias, Michaël Quentin, Yongpan Chen, Janice Almeida-engler, Zhenchuan Mao, Qinghua Sun, Qian Liu, Bingyan Xie, Pierre Abad, et al.

\section{To cite this version:}

Jianlong Zhao, Joffrey Mejias, Michaël Quentin, Yongpan Chen, Janice Almeida-engler, et al.. The root-knot nematode effector MiPDI1 targets a stress-associated protein (SAP) to establish disease in Solanaceae and Arabidopsis. New Phytologist, 2020, 228 (4), pp.1417-1430. 10.1111/nph.16745 . hal-03148658

\section{HAL Id: hal-03148658 \\ https: / hal.inrae.fr/hal-03148658}

Submitted on 8 Dec 2021

HAL is a multi-disciplinary open access archive for the deposit and dissemination of scientific research documents, whether they are published or not. The documents may come from teaching and research institutions in France or abroad, or from public or private research centers.
L'archive ouverte pluridisciplinaire HAL, est destinée au dépôt et à la diffusion de documents scientifiques de niveau recherche, publiés ou non, émanant des établissements d'enseignement et de recherche français ou étrangers, des laboratoires publics ou privés. 
1 The root-knot nematode effector MiPDI1 targets a stress-associated protein, SAP, to

2 establish disease in Solanaceae and Arabidopsis

3

4 Jianlong Zhao ${ }^{1,2,3}$, Joffrey Mejias ${ }^{3}$, Michael Quentin ${ }^{3}$, Yongpan Chen ${ }^{1,3}$, Janice de

$5 \quad$ Almeida-Engler ${ }^{3}$, Zhenchuan $\mathrm{Mao}^{2}$, Qinghua Sun², Qian Liu', Bingyan Xie², Pierre

$6 \operatorname{Abad}^{3}$, Bruno Favery ${ }^{3^{*}}$ and Heng Jian ${ }^{1^{*}}$

7 * co-corresponding authors

8

$9 \quad{ }^{1}$ Department of Plant Pathology and Key Laboratory of Pest Monitoring and Green

10 Management of the Ministry of Agriculture, China Agricultural University, Beijing, China

$11{ }^{2}$ Institute of Vegetables and Flowers, Chinese Academy of Agricultural Science, Beijing, China

12 3'INRAE, Université Côte d'Azur, CNRS, ISA, F-06903 Sophia Antipolis, France

13

$14 *$ Author for correspondence

15 Pr. Heng Jian

16 Yuanmingyuan West Road No. 2, Haidian District, Beijing, China

17 Tel: +8613552481996

18 Email: hengjian@cau.edu.cn

20 Dr. Bruno Favery

21400 route des chappes, BP 167, 0690 Sophia Antipolis, France

22 Email: bruno.favery@inrae.fr

24 Jianlong Zhao zhaojianlong@caas.cn,

25 Joffrey Mejias joffrey.mejias@etu.univ-cotedazur.fr,

26 Michael Quentin michael.quentin@inrae.fr,

27 Yongpan Chen chenyongpan1@163.com,

28 Janice de Almeida-Engler janice.de-almeida@inrae.fr, 
29 Zhenchuan Mao maozhenchuan@,caas.cn,

30 Qinghua Sun sunqinghua h@163.com,

31 Qian Liu liuqian@cau.edu.cn,

32 Bingyan Xie xiebingyan@caas.cn,

33 Pierre Abad pierre.abad@inrae.fr,

34 Bruno Favery bruno.favery@inrae.fr,

35 Heng Jian hengjian@,cau.edu.cn

36

37 Total word count

38 5,651 words (Introduction, 851; Materials and Methods, 1,830; Results, 1,416; Discussion,

39 1,402; Acknowledgements, 151).

40 Figures: 6 (All figures in colour).

41 Supporting information files: 11 (3 tables and 8 figures). 
- Large amounts of effectors are secreted by the oesophageal glands of plant-parasitic nematodes, but their molecular mode of action remains largely unknown. We characterised a Meloidogyne incognita protein disulphide isomerase (PDI)-like effector protein (MiPDI1) that facilitates nematode parasitism. glands of $M$. incognita. It was significantly upregulated during parasitic stages. Immunolocalisation demonstrated MiPDI1 secretion in planta during nematode migration and within the feeding cells. Host-induced silencing of the MiPDI1 gene affected the ability of the nematode to infect the host, whereas MiPDI1 expression in Arabidopsis increased susceptibility to $M$. incognita, providing evidence for a key role of MiPDI1 in $M$. incognita parasitism.

- Yeast two-hybrid assays, BiFC and Co-IP showed that MiPDI1 interacted with a tomato stress-associated protein (S1SAP12) orthologous to the redox-regulated AtSAP12, which plays an important role in plant responses to abiotic and biotic stresses. SAP12 silencing or knocking out in N. benthamiana and Arabidopsis increased susceptibility to M. incognita. acclimation in Solanaceae and Arabidopsis.

64 Key words: plant-parasitic nematodes, effector, stress, redox, giant cells 


\section{Introduction}

Plant parasitic nematodes are among the most economically devastating plant pathogens, causing global yield losses of more than 100 billion dollars each year (Abad et al., 2008). The obligate sedentary endoparasitic nematodes causing the most severe problems are root-knot nematodes (RKNs) and cyst nematodes (CNs). The RKNs, Meloidogyne spp., can infest more than 5,500 crop species (Blok et al., 2008), and therefore represent a huge threat to agricultural production. After hatching, the infective second-stage juveniles (J2s) are attracted to the tip of plant roots. They penetrate the root elongation zone and migrate between cells to reach the vascular cylinder of the plant. There, they become sedentary, and construct a feeding site. This feeding site consists of around seven multinucleate giant cells, resulting from nuclear divisions and isotropic growth, surrounded by cells that divide and initiate vascular differentiation. The giant cells are the sole source of nutrients for the developing nematode (Favery et al., 2016). RKNs ensure that parasitism is successful by secreting a large number of effectors that help juveniles to invade roots, suppress plant defence mechanisms, and induce and maintain giant cells. These effectors are mostly produced in the three oesophageal glands, but they may also be secreted by other organs, such as the amphids and hypodermis (Mejias et al., 2019; Vieira \& Gleason, 2019). The precise localisation of effectors and the identification of their host targets are essential for a better understanding of their biological functions. Only a few host target proteins of RKN effectors have been identified to date (Mejias et al., 2019). The M. incognita effector Mi16D10 has been shown to interact with Arabidopsis SCARECROW-like transcription factors (Huang et al., 2006). The M. graminicola effector MgMO237 has been shown to interact with three rice defense-related proteins (OsGSC, OsCRRSP55 and OsBetvI) (Chen et al., 2018) and the Mg16820 and M. chitwoodi Mc01194 effectors interact with rice dehydration-stress inducible protein 1 (OsDIP1) and Arabidopsis papain-like cysteine protease (RD21A), respectively (Davies et al., 2015; Naalden et al., 2018). A MACROPHAGE MIGRATION INHIBITORY FACTOR-like effector, MiMIF-2, was recently shown to interact with two Arabidopsis annexins, mediating plant immune responses (Zhao et al., 2019). However, the functions of a large number of RKN effectors, their plant targets and working mechanisms remain unknown and in need of clarification. 
95 Protein disulphide isomerase (PDI) is a protein thiol oxidoreductase located in the eukaryotic 96 endoplasmic reticulum. PDI has both protein disulphide isomerase and protein-glutamine gamma-glutamyltransferase activities. It is involved in the oxidoreduction and isomerisation of protein disulphide bonds, peptidyl-proline hydroxylation to 4-hydroxy-L-proline, and protein deglutathionylation (Ali Khan \& Mutus, 2014). More than 50 PDI-like proteins have been identified in fungi, plants, animals and humans. The free-living nematode Caenorhabditis elegans has three conserved PDI-like genes, PDI-1, PDI-2, and PDI-3, and PDI-2 has been shown to be expressed in the hypodermis and cuticle collagen (Winter \& Page, 2000). Many animal parasites, including protozoans in particular, have PDI-like proteins that play major roles in parasitism (Achour et al., 2002; Han et al., 2014). Three PDI-like proteins in the tick Haemaphysalis longicornis have been shown to be expressed predominantly in the salivary glands, and blood feeding significantly increases the expression of HlPDI-1 and HIPDI-3 (Liao et al., 2007). There are also many PDI-like proteins in malaria parasites. Plasmodium falciparum PfPDI-8 has specific enzyme activity and facilitates the disulphide-dependent conformational folding of a malaria protein (Mahajan et al., 2006).

110 Secreted PDIs have also recently been characterised in plant pathogens. The PDI1 protein of 111 Phytophthora parasitica (PpPDI1) is associated with haustoria-like structures and contributes to 112 plant infection (Meng et al., 2015). Two PDI-like effectors have been identified in RKNs and 113 CNs, shown to be expressed in oesophageal glands and upregulated in late parasitic J2s or J3. A 114 functional analysis of Heterodera schachtii HsPDI showed that this protein promoted 115 parasitism by regulating the plant ROS burst (Habash et al., 2017). Recombinant $M$. 116 graminicola MgPDI protein had oxidase and isomerase activities. The expression of both $117 \mathrm{MgPDI}$ and $\mathrm{HsPDI}_{s}$ was induced by exogenous $\mathrm{H}_{2} \mathrm{O}_{2}$, suggesting that PDI may protect 118 nematodes from oxidative stress (Tian et al., 2019). Interestingly, PDI-like proteins were 119 identified in the secretome of $M$. incognita juveniles (Bellafiore et al., 2008) and the proteome 120 of oesophageal gland cells from M. incognita females (Wang et al., 2012), demonstrating that 121 these proteins are secreted by M. incognita. However, the detailed mode of action and plant targets of PDI-like effectors remain unknown. 
In this study, we characterised a new PDI-like gene from M. incognita cDNA (MiPDII) that is

124 expressed in the subventral oesophageal glands. Immunohistochemical staining of tomato root sections with an anti-MiPDI1 antibody showed that this protein was secreted into infected roots.

126 Our findings also showed that MiPDI1 was important for nematode infection. Yeast two-hybrid 127 assays, BiFC and Co-IP showed that MiPDI1 interacted physically with a stress-associated 128 protein (SISAP12) from tomato. Virus-induced gene silencing (VIGS) of orthologues in 129 knockout lines of $N$. benthamiana and Arabidopsis resulted in higher levels of M. incognita 130 infection. Moreover, MiPDI1 expression in planta affects the expression of Arabidopsis 131 defence-associated genes. Our data suggest that MiPDI1 may act as a novel effector, promoting 132 M. incognita parasitism by fine-tuning SAP-mediated host responses.

134 Materials and methods

\section{Nematodes and plant materials}

136 Egg masses of $M$. incognita (Morelos strain) were collected from tomato plants (Solanum 137 lycopersicum cv St Pierre). The hatched preparasitic second-stage juveniles (pre-J2s) were 138 collected for plant inoculation. Various M. incognita stages were isolated from digested tomato 139 roots, as previously described (Zhao et al., 2019). Surface-sterilised Arabidopsis thaliana seeds 140 (ecotype Col-0) were sown on Murashige and Skoog (MS) medium in sterile conditions. After 141 germination, the plantlets were transplanted into pots containing soil and sand (1:1) and grown 142 at $21^{\circ} \mathrm{C}$. The T-DNA mutant line for AtSAP12 (At3g28210) (SALK_014706) was obtained from 143 the Arabidopsis Biological Resource Center (ABRC, USA). Nicotiana benthamiana, Solanum 144 lycopersicum and $N$. tabacum plants were grown at $24^{\circ} \mathrm{C}$ (photoperiod, $16 \mathrm{~h}: 8 \mathrm{~h}$, light : dark).

146 Sequence analysis, alignment and phylogenetic tree

147 MiPDI sequences were obtained from Meloidogyne genomic resources 148 (http://www6.inra.fr/meloidogyne_incognita/). Sequences were aligned with the MAFFT tool 149 on the EBI server (https://www.ebi.ac.uk/Tools/msa/mafft/). The alignment obtained was used 150 as input for the IQTree Web server (http://iqtree.cibiv.univie.ac.at/) (Trifinopoulos et al., 2016), 
151 to generate the maximum likelihood phylogenetic tree. The model chosen by the inbuilt model 152 test was $\mathrm{LG}+\mathrm{F}+\mathrm{I}+\mathrm{G} 4$. Support for the nodes was calculated with a hundred bootstrap replicates.

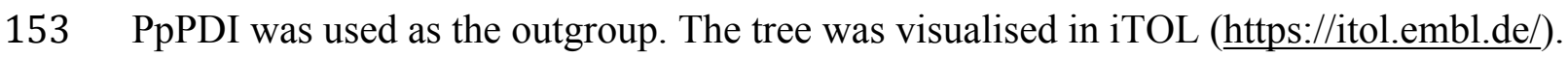

\section{RNA extraction and real-time quantitative RT-qPCR}

A Dynabeads ${ }^{\circledR}$ mRNA DIRECT ${ }^{\mathrm{TM}}$ kit (Invitrogen, USA) was used to extract mRNA from $M$. incognita. Arabidopsis, tomato and tobacco total RNA were extracted with TRIzol Reagent

158 (Invitrogen, USA). We synthesised cDNA with the reverse transcriptase SuperScript III 159 (Invitrogen, USA), in accordance with the manufacturer's instructions. RT-qPCR was 160 performed with an ABI Prism 7000 (Applied Biosystems, USA) real-time PCR system (the 161 primers are shown in Table S1). M. incognita GAPDH (Minc12412) and HK14 (Minc18753) or 162 A. thaliana OXA1 (AT5G62050) and UBP22 (AT5G10790) were used as internal controls for 163 the normalisation of RT-qPCR data. PCR was performed with SYBR Premix Ex Taq (TaKaRa, 164 Japan), as follows: $95^{\circ} \mathrm{C}$ for $30 \mathrm{~s}$, followed by 40 cycles of $95^{\circ} \mathrm{C}$ for $5 \mathrm{~s}, 60^{\circ} \mathrm{C}$ for $31 \mathrm{~s}$, and $16595^{\circ} \mathrm{C}$ for $15 \mathrm{~s}, 60^{\circ} \mathrm{C}$ for $60 \mathrm{~s}$, and $95^{\circ} \mathrm{C}$ for $15 \mathrm{~s}$. Data were analysed by the $2^{-\Delta \Delta \mathrm{Ct}}$ method (Livak $166 \&$ Schmittgen, 2001). Three technical replicates for two to four independent biological experiments were performed.

\section{In situ hybridisation (ISH) of MiPDI1 and immunolocalisation in preparasitic J2s}

ISH was performed on freshly hatched M. incognita preparasitic J2s (pre-J2s), as previously described (Jaouannet et al., 2018). The MiPDI1 was amplified with the specific primers ISHPDI-F and -R (Table S1). Immunolocalisation was performed as previously described (Jaubert

173 et al., 2002; Jaubert et al., 2005). A polyclonal antibody against MiPDI1 was produced with a specific peptide (KEGSPPSENSLDDLVE). Western blotting was performed to check the

175 specificity of this antibody. Immunolocalisation was performed directly on $M$. incognita pre176 parasitic J2s with the anti-MiPDI1 antibody (1:300) and the Alexa Fluor 488-conjugated goat 177 anti-rabbit antibody (1:500) (Molecular Probes, Eugene). As a negative control, nematodes were incubated with pre-immune serum. Images were collected with a confocal microscope 


\section{Immunohistochemistry on tomato gall sections}

182 Tomato galls were collected 5 and 10 days post infection (dpi) with $M$. incognita, cut into small 183 pieces with a razor blade in a Petri dish and fixed in $8 \%$ formaldehyde in $50 \mathrm{mM}$ piperazine-N, $\mathrm{N}$-bis (ethanesulphonic acid) (PIPES) buffer ( $\mathrm{pH}$ 6.9). The immunolocalisation procedure was performed essentially as previously described (de Almeida Engler et al., 2004). Galls were dehydrated and embedded in butyl-methylmethacrylate and sections were incubated in acetone for $30 \mathrm{~min}$ to remove the butyl-methylmethacrylate. Slides containing $5 \mu \mathrm{m}$-sectioned galls were then treated with a series of ethanol solutions and incubated in a blocking solution of $1 \%$ BSA. Sections were subsequently incubated with anti-MiPDI1 antibody (1:300) at $4{ }^{\circ} \mathrm{C}$ overnight and then at $37^{\circ} \mathrm{C}$ for 2 hours in a damp box. As a negative control, gall sections were incubated with pre-immune serum. Finally, slides were incubated with Alexa Fluor 488conjugated goat anti-rabbit antibody (Molecular Probes, Eugene) and cell nuclei were stained with 4', 6-diamidino-2-phenylindole (DAPI) (Sigma, USA). Images were captured at an excitation wavelength of $488 \mathrm{~nm}$ by confocal microscopy (Zeiss LSM880, Germany).

\section{In planta RNAi, DEX-induced MiPDI1 expressing Arabidopsis lines and RKN infection}

MiPDI1 fragments (forward and reverse) were amplified with primers (Table S1) inserted separately into the forward and backward sequences of the pSAT5 intron of the pSuper-RNAi

200 vector (Dafny-Yelin et al., 2007). The MiPDI1 coding sequence (cds) without the signal 201 peptide sequence for secretion (MiPDI1 woSP) was amplified (Table S1) and inserted into the 202 PTA7001 vector (a gift from Professor Wenxian Sun, China Agricultural University) to 203 generate PTA7001-MiPDI1-FLAG. These vectors were used to transform Agrobacterium 204 tumefaciens GV3101, which was in turn used to transform A. thaliana Col-0 (WT) by the floral 205 dip method (Zhang et al., 2006). Homozygous T3 plants from three pdi-Ri lines and two 206 MiPDI-FLAG lines were used. Lines were verified by western blotting after induction with 30 
$207 \mu \mathrm{M}$ dexamethasone (DEX). The homozygous gfp-Ri T3 lines have been described elsewhere 208 (Zhao et al., 2019). Arabidopsis plants were inoculated with 300 pre-J2s of M. incognita. The 209 RNAi effect was assessed 10 dpi by RT-qPCR. Arabidopsis roots were then collected and 210 washed carefully at 35 dpi. Nematodes (parasitic juveniles at any stages and females) were 211 stained by the sodium hypochlorite-acid fuchsin method (Bybd et al., 1983). Nematodes, galls 212 and egg masses were counted under a stereomicroscope microscope (Olympus, Japan). Three 213 independent replicates were performed for each experiment, with counting on 30 plants of each 214 line in each replicate.

\section{Subcellular localisation and bimolecular fluorescence complementation (BiFC)}

217 MiPDI1 woSP was amplified by PCR with specific Gateway primers (Table S1) and inserted 218 into the pDONR207 donor vector. It was inserted into the pK7WGF2 (N-terminus eGFP) 219 vector by recombination, with Gateway technology (Invitrogen). The SlCYP woSP and 220 SISAP12 ORFs were inserted into the pK7WGR2 (N-terminus RFP) vector by recombination. 221 For the BiFC assay, MiPDI1 was inserted into YFPn-, and S1SAP12 and S1CYP were inserted 222 into YFPc-vectors (Walter et al., 2004). The constructs were used to transform A. tumefaciens 223 strain GV3101 or GV3301. Leaves from three- to four-week-old N. tabacum plants were 224 subjected to agroinfiltration with recombinant strains of $A$. tumefaciens, as described by Zhao 225 et al. (2019). Images were captured by confocal microscopy (Zeiss LSM880, Germany) at an 226 excitation wavelength of $488 \mathrm{~nm}$ for GFP or YFP and $561 \mathrm{~nm}$ for RFP.

229 For the $\mathrm{Y} 2 \mathrm{H}$ screens, MiPDI1 woSP or a mutated version, MiPDI1-mu (the first CGHC 230 activation site mutated to CGHG), was amplified (Table S1) and inserted into pB27 as a C231 terminal fusion to LexA. The constructs were verified by sequencing and used to transform a $232 \mathrm{~L} 40 \Delta \mathrm{Ga} 14$ (mat $\alpha$ ) yeast strain. These baits were used to screen a random-primed cDNA library 233 made from tomato roots infected with M. incognita and Ralstonia solanacearum in the Y187 234 (mat $\alpha$ ) yeast strain (Hybrigenics Services, Paris, France), by a mating approach. Diploids 
235 displaying interactions were selected on a medium lacking tryptophan, leucine and histidine.

236 The prey fragments of the positive clones were amplified by PCR and sequenced. The resulting 237 sequences were used to identify the tomato interacting proteins with the Sol Genomics Network 238 (https://solgenomics.net/) blast analysis tools. Pairwise $\mathrm{Y} 2 \mathrm{H}$ assays were conducted following 239 the instruction of Clontech protocol (Clontech, USA). Briefly, the MiPDI1 coding region 240 without the predicted signal peptide sequence was cloned into the pGBKT7 vector as the bait. 241 The sequences encoding the SAP12 (SISAP12, NbSAP12 and AtSAP12) were cloned into the 242 pGADT7 vector as the preys. After co-transformation of yeast (Y2HGOLD) and screening on $243 \mathrm{SD} /$-Leu-Trp plates, positive clones were verified and selected to grow on SD/-Leu-Trp-His 244 medium with $0.5 \mathrm{mM}$ 3-Amino-1,2,4-Triazole (3AT).

245 For Co-IP assays, the MiPDI1, SlSAP12, and SlCYP sequences were inserted into the PVX 246 vector pGR107 with a FLAG-tag fused at the N-terminus (FLAG-MiPDI1) or an HA-tag fused 247 at the C-terminus (S1SAP12-HA and S1CYP-HA) (Zhao et al., 2019) and the resulting 248 constructs were used to transform A. tumefaciens GV3101, which was then used to transform 249 N. tabacum leaves. Total proteins were extracted from N. tabacum leaves. Co-IP was 250 performed with anti-FLAG M2 affinity gel resin (Sigma-Aldrich, USA), according to the 251 manufacturer's instructions. Briefly, the gel resin was washed twice with $1 \mathrm{ml}$ ice-cold PBS. 252 We then immediately added $1 \mathrm{ml}$ of protein solution and incubated the mixture at $4^{\circ} \mathrm{C}$ for $4 \mathrm{~h}$. 253 The resin was thoroughly washed 5 times with $1 \mathrm{ml}$ of ice-cold PBS, and then the proteins were 254 eluted for western blot analysis. Anti-FLAG antibody (1:5000) and anti-HA (1:5000) 255 antibodies (MBL, Japan) were incubated with the blot for protein detection.

\section{Virus-induced gene silencing (VIGS)}

258 VIGS assays were performed on N. benthamiana. The $N$. benthamiana orthologues of SlSAP12 259 and SlCYP were identified in https://solgenomics.net. Regions for targeted gene silencing of 260 NbSAP12_Niben101Scf06280g06001/Niben101Scf06013g06013 and 261 NbCYP_Niben101Scf12813g00004 were identified with the Sol Genomics Network VIGS-Tool 262 (Fernandez-Pozo et al., 2015). Specific fragments were amplified by PCR with the primer pairs 

TRV2-SAP12-F/TRV2-SAP12-R, and TRV2-CYP-F/TRV2-CYP-R (Table S1). The PCR

264 products were digested with EcoRI and XhoI, and ligated to the tobacco rattle virus RNA 2 vector (TRV2) for transformation of the A. tumefaciens strain GV3101. VIGS assays were performed as previously described, by the infiltration of agrobacterial strains containing RNA 1 vector (TRV1) or TRV2 into leaves (Velasquez et al., 2009; Lange et al., 2013). The phytoene desaturase $(P D S)$ gene was used as a positive control to check for successful gene silencing, which results in typical photobleaching symptoms on young growing leaves. Empty TRV2 and TRV1 were used as negative controls. One-week post-agroinfiltration, TRV-infected plants were inoculated with $200 \mathrm{M}$. incognita J2s. One-week later, five plants were used for RT-qPCR to assess the efficacy of gene silencing. Galls and females producing egg masses were counted 27350 dpi.

\section{Protein extraction, SDS-PAGE and western blotting}

Total protein was extracted from agro-infiltrated $N$. tabacum leaves 2 dpi, with a protein extraction kit (Beijing ComWin Biotech Co., Ltd., China), according to the manufacturer's instructions. Briefly, $0.1 \mathrm{~g}$ of plant material was ground in liquid nitrogen and added to $0.5 \mathrm{ml}$ of protein extraction buffer supplemented with the protease inhibitor PMSF (Sigma). Samples were kept on ice for $30 \mathrm{~min}$, centrifuged at $12,000 \mathrm{rpm}$ for $20 \mathrm{~min}$ at $4^{\circ} \mathrm{C}$, and the protein was

281 obtained in the supernatant. Proteins were fractionated by sodium dodecyl sulphate polyacrylamide gel electrophoresis (SDS-PAGE). For western blotting, an anti-MiPDI1 primary antibody was added to TBST (TBS with $0.1 \%$ Tween $20,1 \%$ nonfat dry milk) at a ratio of 1 : 6000. The membrane was then incubated with a secondary antibody directed against GFP 285 (ABclonal, China), a FLAG-HRP antibody or an HA-HRP antibody (MBL, Japan), at a dilution of 1: 10000. Proteins were detected with the eECL Western Blot Kit (Beijing ComWin Biotech Co., Ltd., China).

\section{Statistical analysis}

290 The data were subjected to one-way ANOVA with Dunnett's multiple comparisons test or two- 
291 way ANOVA with Tukey's multiple comparisons test. Statistical computations were carried out

292 using GraphPad Prism (GraphPad Software Inc, La Jolla, CA, USA).

\section{Accession numbers}

295 All accession numbers are provided in Table S2. The GenBank accession numbers of $M$. incognita PDI sequences are MT370326 (MiPDI1), MT370324 (MiPDI2a) and MT370327 (MiPDI2b).

\section{Results}

\section{Identification of secreted M. incognita PDIs}

301 Using M. incognita genome and transcriptome datasets (Abad et al., 2008; Nguyen et al., 2018), 302 we identified MiPDI1 (Minc07853a / MT370326) and MiPDI2 (MiPDI2a / Minc12006 / MT370324 and MiPDI2b / Minc15047 / MT370327, which are 99\% identical). Sequence analysis showed that MiPDI1 and MiPDI2s contained the PDI structures common to other PDI-

305 like proteins: four thioredoxin (Trx) domains (two activation sites in the first and fourth 306 domains) and a N-terminal signal peptide (Fig. 1a). MiPDI1 was identified in previous $M$. 307 incognita J2 secretome and female proteome studies, whereas MiPDI2 was detected only among female oesophageal gland proteins (Bellafiore et al., 2008; Wang et al., 2012).

309 Multiple sequence alignment and phylogenetic analysis were performed with PDI protein 310 sequences from $M$. incognita, M. graminicola (MgPDI), H. schachtii (HsPDI), the free-living 311 nematode Caenorhabditis elegans (CePDI1, CePDI2 and CePDI3), and the animal parasites 312 Brugia malayi (BmPDI) and Phytophthora parasitica PpPDI1 (Fig. 1b, c). Two subgroups were 313 identified in the tree; MiPDI2a/b was found to be more similar to the recently described HsPDI 314 and MgPDI, whereas MiPDI1 formed a subgroup with CePDI1, CePDI2 and BmPDI (Fig. 1c). 315 These results suggest that MiPDI1 is a new PDI-like protein that could potentially act as an 316 effector of $M$. incognita. 


\section{stages of M. incognita}

The distribution of MiPDII transcripts was investigated by ISH in preparasitic M. incognita J2s (pre-J2s). A specific signal was detected in subventral oesophageal gland cells after hybridisation with the digoxigenin-labelled MiPDI1 antisense probe (Fig. 2a; Fig. S1). No signal was detected in pre-J2s with sense negative controls (Fig. 2b).

The transcription of MiPDII was analysed by RT-qPCR at various stages of $M$. incognita development. MiPDII was significantly more strongly expressed in parasitic juveniles (Par-J 5 and 15 dpi) and females (Fig. 2c) than in pre-J2 and eggs. Together, these findings suggest that MiPDII may play an important role in nematode parasitism in the plant.

\section{MiPDI1 is secreted into plant tissues during parasitism}

We performed immunolocalisation to determine whether and where MiPDI1 was secreted in host roots. A specific anti-MiPDI1 antibody was produced and a single band was detected in total protein samples from female pre-J2s, at the expected size of $57 \mathrm{kDa}$, corresponding to MiPDI1 without its signal peptide, and from N. tabacum leaves expressing MiPDI1-FLAG (Fig. $\mathrm{S} 2)$. No signal was detected in protein samples from uninfected tomato roots, tomato leaves, $N$. tabacum and N. benthamiana leaves (Fig. S2). Immunolocalisation showed that the MiPDI protein was present in the subventral glands of pre-J2s (Fig. 2d,e), consistent with the ISH results. Immunohistochemistry on tomato gall sections at 5 and 10 dpi showed that MiPDI1 was present in the parasitic juveniles, at the stylet tip, and in the apoplast at early stages of infection (Fig. 2f,g; Fig. S3). In a number of gall sections containing nematodes, MiPDI1 was detected in both the anterior part of the nematode, along the cell wall of adjacent giant cells and in the giant cells (Fig. 2h,i,j; Fig. S3). No signal was observed in tomato gall sections incubated with preimmune serum (Fig. S3). These results provide evidence for the secretion of the MiPDI1 effector in planta during parasitism.

Host-derived RNAi silencing and ectopic expression of MiPDI1 shows the importance for M. incognita parasitism 
347 We investigated the role of MiPDI1 in M. incognita parasitism, through the use of host-derived 348 RNAi to silence MiPDI1 in feeding nematodes. Three T3 homozygous pdi-Ri Arabidopsis lines 349 (pdi-1, pdi-3 and pdi-5) transformed with the MiPDI1 hairpin dsRNA were obtained and 350 infected with M. incognita. RT-qPCR analysis of MiPDI1 in parasitic juveniles (10 dpi) showed 351 much lower levels of MiPDI1 expression (about 60\%) in the three T3 pdi-Ri lines than in the control (WT and gfp-Ri) lines (Fig. 3a). Four weeks post infection, pdi-Ri lines presented significantly smaller numbers of galls and parasitic nematodes in the roots (Fig. 3b,c).

Two independent Arabidopsis lines ectopically expressing MiPDII were generated, in which expression was induced by $30 \mu \mathrm{M}$ dexamethasone (DEX). MiPDI1-expressing leaves showed some necrosis spots after they were sprayed with DEX (Fig. S4). The susceptibility to nematode infection of these two transgenic Arabidopsis lines was then determined. Both these transgenic lines were more susceptible $(P<0.05)$ to $M$. incognita infection than wild-type Arabidopsis (WT) after 35 dpi (Fig. 3d). Thus, the data for MiPDI1 silencing and overexpression provide evidence for a key role of MiPDI1 in M. incognita parasitism.

\section{MiPDI1 interacts with SISAP12, a stress-associated AN1-type zinc finger protein}

We searched for host proteins interacting with MiPDI1 in two independent yeast two-hybrid analyses. We used the MiPDII CDS without the signal peptide, or a form with a mutated first active site as baits to screen a tomato root cDNA library. In total, 7.5 and 26.7 million interactions were tested, respectively, leading to the identification of 13 proteins interacting with MiPDI1 and six proteins interacting with the mutated form (Table S3). The preys captured

368 several times included two zinc finger proteins, a RING-type protein (Solyc03g026060), the AN1-type Stress-Associated Protein 12 (S1SAP12; Solyc02g087210), and two eukaryotic thiol proteases or cysteine proteases displaying $72.4 \%$ identity (Solyc04g078540 and

Solyc12g088670). Interestingly, the S1SAP12 protein and one cysteine protease

372 (Solyc04g078540, referred to hereafter as SlCYP) were identified in both independent screens, 373 and were thus studied further. Reciprocal BLASTP showed that Solyc02g087210 was an 374 orthologue of AtSAP12. Both these small cysteine-rich proteins (10\%) are AN1-type SAPs, 
375 containing only two AN1 zinc-finger domains, and 16 conserved cysteine residues (Fig. S5).

376 Pairwise yeast two-hybrid assays with full-length proteins confirmed the interaction between 377 SISAP12 or S1CYP and MiPDI1 in yeast (Fig. 4a). Subcellular localisation studies involving 378 agroinfiltration in N. tabacum showed a cytoplasmic localisation of RFP-S1CYP and MiPDI1379 GFP and a nucleo-cytoplasmic localisation of RFP-S1SAP12 in epidermal cells (Fig. 4b). We 380 also investigated the interaction of MiPDI1 with SICYP and S1SAP12 in planta in bimolecular 381 fluorescence complementation (BiFC) assays. The co-expression of YFPn-MiPDI1 and 382 SISAP12-YFPc reconstituted YFP fluorescence signals in the cytoplasm of $N$. tabacum 383 epidermal cells (Fig. 4c, Fig. S6). We also performed co-immunoprecipitation (Co-IP) experiments to check the interaction between MiPDI1 and SICYP or SISAP12. MiPDI1 385 interacted with S1SAP12 in N. tabacum, further confirming the association of these two proteins 386 in planta (Fig. 4d). By contrast, no interaction was detected between MiPDI1 and SICYP, by 387 either BiFC or Co-IP (Fig. 4c,d). These results confirm the specific interaction of MiPDI1 with SISAP12 in plant cells.

\section{SAP12 affects $M$. incognita parasitism}

391 We further investigated the role of SAP12 in mediating the response to M. incognita, by 392 silencing the $N$. benthamiana SAP12 genes (Niben101Scf06280g06001 and 393 Niben101Scf06013g06013 named NbSAP12s) or CYP (Niben101Scf12813g00004, named $394 \mathrm{NbCYP}$ ) by virus-induced gene silencing (VIGS) (Table S2, Fig. S7). Quantitative RT-PCR analysis of the expression of the targeted homologous genes showed that NbSAP12s and $396 N b C Y P$ were downregulated (55-60\%) in the plants subjected to VIGS relative to control plants 397 (Fig. 5a; Fig. S7). NbSAP12 silencing led to an increase in the number of females producing egg masses relative to the empty TRV2 control, whereas no significant effect was observed 399 with $N b C Y P$ silencing (Fig. 5b). Finally, we tested a knockout T-DNA insertional mutant for 400 AtSAP12 (AT3G28210) (Fig. S8). The number of females producing egg masses and the 401 number of galls were larger in homozygous Arabidopsis knockout (KO) sap12 plants 402 (Salk_014706) than in wild-type plants (Fig. 5c). These results indicate that SAP12 proteins 
may play a role in plant - M. incognita interaction in Solanaceae and Arabidopsis.

\section{MiPDI1 expression in Arabidopsis affects stress- and defence-associated gene expression}

406 We investigated the effects of MiPDII expression and sap12 mutation on stress tolerance in 407 Arabidopsis. We analysed genes involved in antioxidative functions (AtCSD1 and AtCSD2; (Ma et al., 2015), and responses to abiotic (AtCOR47, AtRAB18 and AtDH1; (Kothari et al., 2016) and biotic (AtEM6, AtPRla, AtPDF1.2a, AtPR4 and AtPR1a; (Qiu et al., 2008; Aslam et al.,

410 2009; Rodiuc et al., 2016; Kang et al., 2017; Chang et al., 2018) stresses in Arabidopsis. Four 411 genes (AtCSD2, AtEM6, AtRAB18 and the salicylic acid (SA) marker AtPRIa) were expressed 412 less strongly in MiPDI1-overexpressing Arabidopsis plants, whereas the levels of expression of 413 jasmonate (JA) and the ethylene markers PDF1.2a and PR4 were much higher in these plants 414 (Fig. 5d). The expression of six genes (AtCSD1, AtCSD2, AtDH1, AtPDF1.2a, AtPR4 and AtPRla) was upregulated in the sap12 KO line, whereas the stress-related genes AtCOR47, AtRAB18 and AtEM6 were downregulated (Fig. 5d). These results suggest that both MiPDI and its target may be responsible for regulating the expression of genes involved in stress responses 418 to RKNs.

\section{Discussion}

421 The repertoire of putative nematode effectors is extremely large, and these molecules have been 422 shown to manipulate many host plant functions to orchestrate the suppression of plant defences 423 and the formation of specialised feeding cells (Mejias et al., 2019). However, few data are 424 available concerning the functions of effectors and few plant targets have been characterised, 425 particularly for RKN effectors. Analyses of the secretomes of plant-parasitic nematodes and 426 animal-parasitic nematodes have provided compelling evidence for the secretion of redox427 regulated proteins, such as Trx, glutathione peroxidases, glutathione-S-transferases and PDIs 428 (Bellafiore et al., 2008; Hewitson et al., 2008). PDIs are involved in the oxidoreduction and 429 isomerisation of protein disulphide bonds, hydroxylation and protein deglutathionylation (Selles 430 et al., 2011; Ali Khan \& Mutus, 2014). Recent studies have shown that H. schachtii and M. 
431 graminicola PDI genes are expressed in the subventral glands of preparasitic J2s and 432 upregulated in parasitic J2s. Functional studies have shown that these PDIs, which belong to the 433 same subgroup as MiPDI2, play important roles in nematode parasitism through ROS 434 detoxification (Habash et al., 2017; Tian et al., 2019). We characterised the role of a secreted 435 M. incognita MiPDI1 effector identified in J2 secretome (Bellafiore et al., 2008; Hewitson et al., 2008) and identified its target in plant-RKN interactions, a stress-associated protein.

\section{MiPDI1 is secreted throughout parasitism and targets the giant cells in planta}

MiPDI1 transcript abundance increased significantly throughout parasitic stages in planta (from juveniles to females). We demonstrated that MiPDI1 was produced in the subventral oesophageal gland and secreted in planta, both in the apoplast during nematode migration, but also within the giant cells. Although the SvGs have been shown to be more active in the early stage of parasitism (Davis et al., 2000), SvGs remain active in planta and produce effectors showing an increase in transcript abundance in parasitic juvenile stages compared to preparasitic J2s (Nguyen et al., 2018). The secretion of MiPDI1 by SvGs would thus allow its production from the migration step to the formation of giant cells. Recent studies showed two PDI-like proteins of PPNs, MgPDI and HsPDI, were localised to the apoplast when GFP fusions were transiently expressed in N. benthamiana (Habash et al., 2017; Tian et al., 2019), indicating their different functional mechanism during nematode parasitism. Despite the hundreds of effectors characterised (Mejias et al., 2019), few RKN effectors have been demonstrated to be secreted in planta and to target giant cells. Example of effectors are the $M$. incognita Mi-EFF1 (Jaouannet et al., 2012) and MiMIF-2 (Zhao et al., 2019), the M. javanica MjNULG1a (Lin et al., 2016) and the M. graminicola MgGPP (Chen et al., 2017) and 455 affected the number of galls and egg masses obtained and delayed nematode development. 456 Accordingly, ectopic MiPDI1 expression in Arabidopsis increased susceptibility to $M$. 457 incognita. These results provide evidence that MiPDI1 is a novel plant-parasitic effector playing an essential role in nematode parasitism. In light of essential roles of PDI-like proteins 
459

460

in redox regulation and mediating pathogens entry in infectious disease (Parakh \& Atkin, 2015), MiPDI1 may protect parasitic nematode stages and feeding cells from oxidative stress. Indeed, studies of antioxidant molecule depletion have shown that the control of plant cell redox status is a key regulator of giant cell effectiveness (Baldacci-Cresp et al., 2012).

\section{MiPDI1 targets the redox-regulated stress-associated SAP12 proteins in Arabidopsis and}

\section{Solanaceae}

We further investigated the function of MiPDI1 in host cells, by searching for the proteins interacting with MiPDI1 in tomato. The putative targets identified included cysteine proteases and stress-associated zinc finger proteins, the activities of which are regulated by thiols/cysteines. Interestingly, the two cysteine proteases (S1CYPs) displaying high levels of sequence identity captured in our Y2H screen are orthologous to Arabidopis RD21a, a known target of the M. chitwoodi effector Mc01194 (Davies et al., 2015). Cysteine proteases have a thiol group in the active site of the enzyme and are known to interact with Trx proteins (Montrichard et al., 2009). PDI-like proteins have been shown to regulate RD21a activity in Arabidopsis (Andeme Ondzighi et al., 2008), but we were unable to confirm the interaction of MiPDI1 with SICYP in plant cells. The silencing of NbCYP in N. benthamiana did not affect plant susceptibility to $M$. incognita, suggesting that these molecules are not functional targets of MiPDI1.

The interaction of MiPDI1 with the tomato stress-associated protein S1SAP12, an AN1-type zinc finger protein was confirmed by $\mathrm{Y} 2 \mathrm{H}, \mathrm{BiFC}$ in planta and co-immunoprecipitation experiments. SISAP12 has been shown to be upregulated at later stages of gall formation in tomato, in response to $M$. incognita attack (Shukla et al., 2018). The cysteine residues of zinc finger proteins are involved in zinc binding. The association of cysteine residues with zinc may therefore be affected by Trx, with consequences for protein activity (Carter \& Ragsdale, 2014). AtSAP12 is a protein that undergoes major reversible redox-dependent conformational changes, facilitating a rapid response to changing environmental conditions (Stroher et al., 2009). Under oxidising conditions $\left(\mathrm{H}_{2} \mathrm{O}_{2}\right)$, oxidised SAP12 forms high-molecular mass aggregates. By 
contrast, DTT and Trx reduce the oligomeric/dimeric form of SAP12 to the monomeric form 488 lacking intermolecular disulphide bridges. SAP12 acts as a redox sensor capable of undergoing changes in its oligomeric conformation as a function of cellular redox potential, thereby transmitting redox information to other cell components (Stroher et al., 2009). MiPDI1, which contains two Trx domains, could potentially regulate the activity of SAP12 by controlling its oligomerisation state in planta.

SAP proteins play important roles in plant responses to abiotic and biotic stresses

SAPs, which contain the AN1 and/or A20 zinc-finger domains in rice, are known to respond rapidly to diverse abiotic stresses and to play important roles in plant responses to these stresses (Krishna et al., 2003; Vij \& Tyagi, 2008; Solanke et al., 2009; Stroher et al., 2009; Dixit et al., 2018). Thirteen SAP genes have been described in tomato (Solanke et al., 2009), 14 in Arabidopsis (Stroher et al., 2009), 18 in rice (Vij \& Tyagi, 2006) and 57 in Brassica napus (He et al., 2019). Levels of AtSAP12 and SISAP12 expression increase immediately in response to 501 various abiotic stresses (Solanke et al., 2009). Interestingly, the Arabidopsis microRNA 502 miR408, a key component of abiotic stress responses, is upregulated at 7 and 14 dpi in galls 503 induced by M. incognita (Medina et al., 2018). Higher levels of miR408 expression are associated with better tolerance to oxidative stress (Ma et al., 2015). Cellular antioxidant

505 capacity is enhanced in plants with high levels of miR408 expression, as demonstrated by the 506 lower levels of reactive oxygen species and the induction of genes associated with antioxidative 507 functions, such as SAP12 (Ma et al., 2015). In rice, OsiSAP8 confers tolerance to abiotic 508 stresses (Kanneganti \& Gupta, 2008). Likewise, OsSAP1 plays important roles in the responses 509 to both abiotic and biotic stresses, by interacting with aminotransferase (OsAMTR1) and the 510 Pathogenesis-Related 1a Protein (OsSCP) (Tyagi et al., 2014; Kothari et al., 2016). AtSAP9 has 511 been shown to mediate ABA signalling in response to biotic and abiotic stresses, possibly via 512 the proteasome pathway (Kang et al., 2017). Meanwhile, the plant A20-AN1 protein acts as a 513 key hub, mediating antiviral immunity (Chang et al., 2018). However, the modes of action of 514 AN1-type proteins in plant pathogen responses remain largely unknown. 
515 SISAP3 and SISAP4 have recently been shown to be positive regulators of immunity, to 516 Pseudomonas syringae pv. tomato (Goldberger et al.) and Botrytis cinerea (Liu et al., 2019a; 517 Liu et al., 2019b). S1SAP3 silencing decreased the Pst DC3000-induced expression of SA 518 signaling and defense genes and attenuated immunity to Pst DC3000, whereas S1SAP3 519 overexpression in transgenic tomato increased them. We show here that SAP12 silencing or 520 knocking out SAP12, in N. benthamiana and in the sap12 Arabidopsis mutant, respectively 521 increased susceptibility to $M$. incognita. Moreover, the expression of some stress-associated 522 marker genes was decreased in MiPDIl-expressing lines, whereas most of the genes 523 investigated were upregulated in the sap12 mutant. Thus, a dual function of MiPDI1 can 524 hypothesized during plant-nematode interaction. MiPDI1 may contribute to $M$. incognita 525 parasitism by protecting nematodes from oxidative stress during migration in planta and by 526 interacting with SAP12 in the giant cells to fine-tune SAP12-mediated responses at the interface 527 of redox signalling, defence and stress acclimation. One of the challenges for the future will be 528 the establishment of assays for investigating the regulatory mechanism and showing how 529 MiPDI1 and SAP12 orchestrate downstream responses.

\section{Acknowledgments}

532 We wish to thank Hybrigenics Services (Paris, France) for providing the pB27 and pP6 vectors, 533 and the L40 $\Delta$ Gal4 and Y187 yeast strains, Dr Antony P. Page (University of Glasgow, UK) for 534 providing an anti-CePDI-2 antibody we used in preliminary experiments and INRA SPE for 535 financing $\mathrm{Y} 2 \mathrm{H}$ library. J.M. benefits from a doctoral fellowship of the Ministère de

536 l'Enseignement Supérieur, de la Recherche et de l'Innovation (MENRT grant). P.A., B.F., and 537 M.Q. are supported by INRA and by the French Government (National Research Agency, 538 ANR) through the 'Investments for the Future' LabEx SIGNALIFE: program reference \#ANR539 11-LABX-0028-01. This research supported by supported by the National Key Research and 540 Development Program of China (No. 2017YFD0200601), the National Natural Science 541 Foundation of China (Nos. 31571987, 31772138), and the National Basic Research Program of 542 China (No. 2013CB127501). J.L.Z and C.Y.P got scholarships from China Scholarship Council 
(No. 201606350083 and No. 201806350108) for studying in INRA, France.

\section{Author contributions}

JZ, JM and MQ designed, performed experiments and analysed the data. YC, ZM, QS, QL, BX performed experiments. JAE gave guidance for immunostaining. MQ, BX and PA supervised some of this work and provided input and expertise. HJ and BF were responsible for the development and guidance of the project. JZ, MQ, PA, BF and HJ wrote the manuscript with input from all co-authors.

\section{References}

Abad P, Gouzy J, Aury JM, Castagnone-Sereno P, Danchin EG, Deleury E, PerfusBarbeoch L, Anthouard V, Artiguenave F, Blok VC, et al. 2008. Genome sequence of the metazoan plant-parasitic nematode Meloidogyne incognita. Nature Biotechnology 26(8): 909-915.

Achour YB, Chenik M, Louzir H, Dellagi K. 2002. Identification of a Disulfide Isomerase Protein of Leishmania major as a Putative Virulence Factor. Infection and Immunity 70(7): 3576-3585.

Ali Khan H, Mutus B. 2014. Protein disulfide isomerase a multifunctional protein with multiple physiological roles. Frontiers in Chemistry 2: 70.

Andeme Ondzighi C, Christopher DA, Cho EJ, Chang SC, Staehelin LA. 2008. Arabidopsis protein disulfide isomerase-5 inhibits cysteine proteases during trafficking to vacuoles before programmed cell death of the endothelium in developing seeds. Plant Cell 20(8): 2205-2220.

Aslam SN, Erbs G, Morrissey KL, Newman MA, Chinchilla D, Boller T, Molinaro A, Jackson RW, Cooper RM. 2009. Microbe-associated molecular pattern (MAMP) signatures, synergy, size and charge: influences on perception or mobility and host defence responses. Molecular Plant Pathology 10(3): 375-387.

Baldacci-Cresp F, Chang C, Maucourt M, Deborde C, Hopkins J, Lecomte P, Bernillon S, Brouquisse R, Moing A, Abad P, et al. 2012. (Homo)glutathione deficiency impairs root-knot nematode development in Medicago truncatula. PLoS Pathogens 8(1): e1002471.

Bellafiore S, Shen Z, Rosso MN, Abad P, Shih P, Briggs SP. 2008. Direct identification of the Meloidogyne incognita secretome reveals proteins with host cell reprogramming potential. PLoS Pathogens 4(10): e1000192.

Blok VC, Jones JT, Phillips MS, Trudgill DL. 2008. Parasitism genes and host range disparities in biotrophic nematodes: the conundrum of polyphagy versus specialisation. Bioessays 30(3): 249-259. 
Bybd DW, Kirkpatrick T, Barker KR. 1983. An improved technique for clearing and staining plant tissues for detection of nematodes. Journal of Nematology 15(1): 142-143.

Carter EL, Ragsdale SW. 2014. Modulation of nuclear receptor function by cellular redox poise. Journal of Inorganic Biochemistry 133: 92-103.

Chang L, Chang HH, Chang JC, Lu HC, Wang TT, Hsu DW, Tzean Y, Cheng AP, Chiu YS, Yeh HH. 2018. Plant A20/AN1 protein serves as the important hub to mediate antiviral immunity. PLoS Pathogens 14(9): e1007288.

Chen J, Hu L, Sun L, Lin B, Huang K, Zhuo K, Liao J. 2018. A novel Meloidogyne graminicola effector, MgMO237, interacts with multiple host defence-related proteins to manipulate plant basal immunity and promote parasitism. Molecular Plant Pathology.

Chen J, Lin B, Huang Q, Hu L, Zhuo K, Liao J. 2017. A novel Meloidogyne graminicola effector, MgGPP, is secreted into host cells and undergoes glycosylation in concert with proteolysis to suppress plant defenses and promote parasitism. PLoS Pathogens 13(4): e1006301.

Dafny-Yelin M, Chung SM, Frankman EL, Tzfira T. 2007. pSAT RNA interference vectors: a modular series for multiple gene down-regulation in plants. Plant Physiology 145(4): 1272-1281.

Davies LJ, Elling AA, Zhang L. 2015. The Arabidopsis thaliana papain-like cysteine protease RD21 interacts with a root-knot nematode effector protein. Nematology 17(6): 655-666.

Davis EL, Hussey RS, Baum TJ, Bakker J, Schots A, Rosso MN, Abad P. 2000. Nematode Parasitism Genes. Annual Review of Phytopathology 38: 365-396.

de Almeida Engler J, Van Poucke K, Karimi M, De Groodt R, Gheysen G, Engler G, Gheysen G. 2004. Dynamic cytoskeleton rearrangements in giant cells and syncytia of nematode-infected roots. The Plant Journal 38(1): 12-26.

Dixit A, Tomar P, Vaine E, Abdullah H, Hazen S, Dhankher OP. 2018. A stress-associated protein, AtSAP13, from Arabidopsis thaliana provides tolerance to multiple abiotic stresses. Plant, Cell \& Environment 41(5): 1171-1185.

Favery B, Quentin M, Jaubert-Possamai S, Abad P. 2016. Gall-forming root-knot nematodes hijack key plant cellular functions to induce multinucleate and hypertrophied feeding cells. Journal of Insect Physiology 84: 60-69.

Fernandez-Pozo N, Menda N, Edwards JD, Saha S, Tecle IY, Strickler SR, Bombarely A, Fisher-York T, Pujar A, Foerster H, et al. 2015. The Sol Genomics Network (SGN)-from genotype to phenotype to breeding. Nucleic Acids Research 43(Database issue): D1036-1041.

Goldberger RF, Epstein CJ, Anfinsen CB. 1963. Acceleration of reactivation of reduced bovine pancreatic ribonuclease by a microsomal system from rat liver. Journal of Biological Chemistry 238: 628-635.

Habash SS, Sobczak M, Siddique S, Voigt B, Elashry A, Grundler FMW. 2017. Identification and characterization of a putative protein disulfide isomerase (HsPDI) as an alleged effector of Heterodera schachtii. Scientific Reports 7(1): 13536.

Han H, Dong H, Zhu S, Zhao Q, Jiang L, Wang Y, Li L, Wu Y, Huang B. 2014. Molecular characterization and analysis of a novel protein disulfide isomerase-like protein of 
Eimeria tenella. PloS One 9(6): e99914.

He X, Xie S, Xie P, Yao M, Liu W, Qin L, Liu Z, Zheng M, Liu H, Guan M, et al. 2019. Genome-wide identification of stress-associated proteins (SAP) with A20/AN1 zinc finger domains associated with abiotic stresses responses in Brassica napus. Environmental and Experimental Botany 165: 108-119.

Hewitson JP, Harcus YM, Curwen RS, Dowle AA, Atmadja AK, Ashton PD, Wilson A, Maizels RM. 2008. The secretome of the filarial parasite, Brugia malayi: proteomic profile of adult excretory-secretory products. Molecular and Biochemical Parasitology 160(1): 8-21.

Huang G, Dong R, Allen R, Davis EL, Baum TJ, Hussey RS. 2006. A root-knot nematode secretory peptide functions as a ligand for a plant transcription factor. Molecular PlantMicrobe Interactions 19(5): 463-470.

Jaouannet M, Nguyen C-N, Quentin M, Jaubert-Possamai S, Rosso M-N, Favery B. 2018. In situ Hybridization (ISH) in Preparasitic and Parasitic Stages of the Plant-parasitic Nematode Meloidogyne spp. Bio-Protocol 8(6).

Jaouannet M, Perfus-Barbeoch L, Deleury E, Magliano M, Engler G, Vieira P, Danchin EG, Da Rocha M, Coquillard P, Abad P, et al. 2012. A root-knot nematode-secreted protein is injected into giant cells and targeted to the nuclei. New Phytologist 194(4): 924-931.

Jaubert S, Ledger TN, Laffaire JB, Piotte C, Abad P, Rosso MN. 2002. Direct identification of stylet secreted proteins from root-knot nematodes by a proteomic approach. Molecular and Biochemical Parasitology 121(2): 205-211.

Jaubert S, Milac AL, Petrescu AJ, de Almeida-Engler J, Abad P, Rosso MN. 2005. In planta secretion of a calreticulin by migratory and sedentary stages of root-knot nematode. Molecular Plant-Microbe Interactions 18(12): 1277-1284.

Kang M, Lee S, Abdelmageed H, Reichert A, Lee HK, Fokar M, Mysore KS, Allen RD. 2017. Arabidopsis stress associated protein 9 mediates biotic and abiotic stress responsive ABA signaling via the proteasome pathway. Plant, Cell \& Environment 40(5): $702-716$.

Kanneganti V, Gupta AK. 2008. Overexpression of OsiSAP8, a member of stress associated protein (SAP) gene family of rice confers tolerance to salt, drought and cold stress in transgenic tobacco and rice. Plant Molecular Biology 66(5): 445-462.

Kothari KS, Dansana PK, Giri J, Tyagi AK. 2016. Rice Stress Associated Protein 1 (OsSAP1) Interacts with Aminotransferase (OsAMTR1) and Pathogenesis-Related 1a Protein (OsSCP) and Regulates Abiotic Stress Responses. Frontiers in Plant Science 7: 1057.

Krishna SS, Majumdar I, Grishin NV. 2003. Structural classification of zinc fingers: survey and summary. Nucleic Acids Research 31(2): 532-550.

Lange M, Yellina AL, Orashakova S, Becker A. 2013. Virus-induced gene silencing (VIGS) in plants: an overview of target species and the virus-derived vector systems. Methods in Molecular Biology 975: 1-14.

Li Y, Williams B, Dickman M. 2017. Arabidopsis B-cell lymphoma2 (Bcl-2)-associated 
664

665

666

667

668

669

670

671

672

673

674

675

676

677

678

679

680

681

682

683

684

685

686

687

688

689

690

691

692

693

694

695

696

697

698

699

700

701

702

703

704

705

athanogene 7 (BAG7)-mediated heat tolerance requires translocation, sumoylation and binding to WRKY29. New Phytol 214(2): 695-705.

Liao M, Hatta T, Umemiya R, Huang P, Jia H, Gong H, Zhou J, Nishikawa Y, Xuan X, Fujisaki K. 2007. Identification of three protein disulfide isomerase members from Haemaphysalis longicornis tick. Insect Biochemistry and Molecular Biology 37(7): 641654.

Lin B, Zhuo K, Chen S, Hu L, Sun L, Wang X, Zhang LH, Liao J. 2016. A novel nematode effector suppresses plant immunity by activating host reactive oxygen speciesscavenging system. New Phytologist 209(3): 1159-1173.

Liu S, Wang J, Jiang S, Wang H, Gao Y, Zhang H, Li D, Song F. 2019a. Tomato S1SAP3, a member of the stress-associated protein family, is a positive regulator of immunity against Pseudomonas syringae pv. tomato DC3000. Molecular Plant Pathology 20(6): 815-830.

Liu S, Yuan X, Wang Y, Wang H, Wang J, Shen Z, Gao Y, Cai J, Li D, Song F. 2019 b. Tomato Stress-Associated Protein 4 Contributes Positively to Immunity Against Necrotrophic Fungus Botrytis cinerea. Molecular Plant-Microbe Interactions 32(5): 566-582.

Livak KJ, Schmittgen TD. 2001. Analysis of relative gene expression data using real-time quantitative PCR and the 2(-Delta Delta C(T)) Method. Methods 25(4): 402-408.

Ma C, Burd S, Lers A. 2015. $m i R 408$ is involved in abiotic stress responses in Arabidopsis. the Plant Journal 84(1): 169-187.

Mahajan B, Noiva R, Yadava A, Zheng H, Majam V, Mohan KV, Moch JK, Haynes JD, Nakhasi H, Kumar S. 2006. Protein disulfide isomerase assisted protein folding in malaria parasites. International Journal for Parasitology 36(9): 1037-1048.

Medina C, da Rocha M, Magliano M, Raptopoulo A, Marteu N, Lebrigand K, Abad P, Favery B, Jaubert-Possamai S. 2018. Characterization of siRNAs clusters in Arabidopsis thaliana galls induced by the root-knot nematode Meloidogyne incognita. BMC Genomics 19(1): 943.

Mejias J, Truong NM, Abad P, Favery B, Quentin M. 2019. Plant Proteins and Processes Targeted by Parasitic Nematode Effectors. Frontiers in Plant Science 10: 970.

Meng Y, Zhang Q, Zhang M, Gu B, Huang G, Wang Q, Shan W. 2015. The protein disulfide isomerase 1 of Phytophthora parasitica (PpPDI) is associated with the haustoria-like structures and contributes to plant infection. Frontiers in Plant Science 6: 632.

Montrichard F, Alkhalfioui F, Yano H, Vensel WH, Hurkman WJ, Buchanan BB. 2009. Thioredoxin targets in plants: the first 30 years. Journal of Proteomics 72(3): 452-474.

Naalden D, Haegeman A, de Almeida-Engler J, Birhane Eshetu F, Bauters L, Gheysen G. 2018. The Meloidogyne graminicola effector $\mathrm{Mg} 16820$ is secreted in the apoplast and cytoplasm to suppress plant host defense responses. Molecular Plant Pathology 19(11): 2416-2430.

Nguyen CN, Perfus-Barbeoch L, Quentin M, Zhao J, Magliano M, Marteu N, Da Rocha M, Nottet N, Abad P, Favery B. 2018. A root-knot nematode small glycine and 

cysteine-rich secreted effector, MiSGCR1, is involved in plant parasitism. New Phytologist 217(2): 687-699.

Parakh S, Atkin JD. 2015. Novel roles for protein disulphide isomerase in disease states: a double edged sword? Frontiers in Cell and Developmental Biology 3: 30.

Qiu JL, Zhou L, Yun BW, Nielsen HB, Fiil BK, Petersen K, Mackinlay J, Loake GJ, Mundy J, Morris PC. 2008. Arabidopsis mitogen-activated protein kinase kinases MKK1 and MKK2 have overlapping functions in defense signaling mediated by MEKK1, MPK4, and MKS1. Plant Physiology 148(1): 212-222.

Rodiuc N, Barlet X, Hok S, Perfus-Barbeoch L, Allasia V, Engler G, Seassau A, Marteu N, de Almeida-Engler J, Panabieres F, et al. 2016. Evolutionarily distant pathogens require the Arabidopsis phytosulfokine signalling pathway to establish disease. Plant, Cell \& Environment 39(7): 1396-1407.

Selles B, Jacquot JP, Rouhier N. 2011. Comparative genomic study of protein disulfide isomerases from photosynthetic organisms. Genomics 97(1): 37-50.

Shukla N, Yadav R, Kaur P, Rasmussen S, Goel S, Agarwal M, Jagannath A, Gupta R, Kumar A. 2018. Transcriptome analysis of root-knot nematode (Meloidogyne incognita)-infected tomato (Solanum lycopersicum) roots reveals complex gene expression profiles and metabolic networks of both host and nematode during susceptible and resistance responses. Molecular Plant Pathology 19(3): 615-633.

Solanke AU, Sharma MK, Tyagi AK, Sharma AK. 2009. Characterization and phylogenetic analysis of environmental stress-responsive SAP gene family encoding A20/AN1 zinc finger proteins in tomato. Molecular Genetics and Genomics 282(2): 153-164.

Stroher E, Wang XJ, Roloff N, Klein P, Husemann A, Dietz KJ. 2009. Redox-dependent regulation of the stress-induced zinc-finger protein SAP12 in Arabidopsis thaliana. Molecular Plant 2(2): 357-367.

Tian ZL, Wang ZH, Maria M, Qu N, Zheng JW. 2019. Meloidogyne graminicola protein disulfide isomerase may be a nematode effector and is involved in protection against oxidative damage. Scientific Reports 9(1): 11949.

Trifinopoulos J, Nguyen LT, von Haeseler A, Minh BQ. 2016. W-IQ-TREE: a fast online phylogenetic tool for maximum likelihood analysis. Nucleic Acids Research 44(W1): W232-235.

Tyagi H, Jha S, Sharma M, Giri J, Tyagi AK. 2014. Rice SAPs are responsive to multiple biotic stresses and overexpression of OsSAP1, an A20/AN1 zinc-finger protein, enhances the basal resistance against pathogen infection in tobacco. Plant Science 225: 68-76.

Velasquez AC, Chakravarthy S, Martin GB. 2009. Virus-induced gene silencing (VIGS) in Nicotiana benthamiana and tomato. Jove-Journal of Visualized Experiments(28).

Vieira P, Gleason C. 2019. Plant-parasitic nematode effectors - insights into their diversity and new tools for their identification. Current Opinion in Plant Biology 50: 37-43.

Vij S, Tyagi AK. 2006. Genome-wide analysis of the stress associated protein (SAP) gene family containing A20/AN1 zinc-finger(s) in rice and their phylogenetic relationship with Arabidopsis. Molecular Genetics and Genomics 276(6): 565-575. 
Vij S, Tyagi AK. 2008. A20/AN1 zinc-finger domain-containing proteins in plants and animals represent common elements in stress response. Functional \& Integrative Genomics 8(3): 301-307.

Walter M, Chaban C, Schutze K, Batistic O, Weckermann K, Nake C, Blazevic D, Grefen C, Schumacher K, Oecking C, et al. 2004. Visualization of protein interactions in living plant cells using bimolecular fluorescence complementation. The Plant Journal 40(3): 428-438.

Wang XR, Moreno YA, Wu HR, Ma C, Li YF, Zhang JA, Yang C, Sun S, Ma WJ, Geary TG. 2012. Proteomic profiles of soluble proteins from the esophageal gland in female Meloidogyne incognita. International Journal for Parasitology 42(13-14): 1177-1183.

Winter AD, Page AP. 2000. Prolyl 4-hydroxylase is an essential procollagen-modifying enzyme required for exoskeleton formation and the maintenance of body shape in the nematode Caenorhabditis elegans. Molecular and Cellular Biology 20(11): 4084-4093.

Zhang X, Henriques R, Lin SS, Niu QW, Chua NH. 2006. Agrobacterium-mediated transformation of Arabidopsis thaliana using the floral dip method. Nature Protocols 1(2): 641-646.

Zhao J, Li L, Liu Q, Liu P, Li S, Yang D, Chen Y, Pagnotta S, Favery B, Abad P, et al. 2019. A MIF-like effector suppresses plant immunity and facilitates nematode parasitism by interacting with plant annexins. Journal of Experimental Botany 70(20): 5943-5958. 
Fig. 1 Primary structure of MiPDIs. (a) Functional domains of MiPDI1 and MiPDI2. MiPDI1 and MiPDI2 have an N-terminal signal peptide for secretion, four thioredoxin domains (a, b, b', a') predicted by an NCBI conserved domain search, and two catalytic domains containing characteristic CGHC active sites in the a and a' domains. (b) A ClustalW2 alignment of the PDI-like proteins MiPDI1 and MiPDI2 (from Meloidogyne incognita), CePDI (Caenorhabditis elegans), HsPDI (Heterodera schachtii), MgPDI (Meloidogyne graminicola), BmPDI (Brugia malayi) and PpPDI (Phytophthora parasitica). Identical and highly similar (>75\%) amino-acid residues are highlighted against black background shading, similar ( $>50 \%)$ amino-acid residues are shown in grey. Yellow background shading indicates the peptide used in the production of the polyclonal anti-MiPDI1 antibody. The sequences for active site CGHC motif are shown in the red frame. The four thioredoxin (TRX) domains are indicated. (c) Maximum likelihood phylogenetic tree of PDI sequences presented in (b). Support for the nodes was calculated with a hundred bootstrap replicates. PpPDI was used as the outgroup.

Fig. 2 Localisation and temporal expression pattern of MiPDI. (a) In situ hybridisation (ISH) of digoxigenin-labelled antisense MiPDII probe to pre-parasitic $M$. incognita J2s, showing transcripts expressed in the subventral oesophageal gland (SvG). The stylet and the metacarpus are indicated. (b) ISH with the MiPDII sense probe gave no signal. (c) Temporal pattern of MiPDI1 expression. The relative level of MiPDII expression throughout nematode development was quantified by RT-qPCR for five different $M$. incognita stages relative to the egg stage (means $\pm \mathrm{SE}$ ). Data were analysed by the $2^{-\Delta \Delta \mathrm{Ct}}$ method. Different letters indicate statistically significant difference between each column using two-way ANOVA following Tukey’s multiple comparisons test $(P<0.05)$. Pre-J2: preparasitic second-stage juvenile; par-J: parasitic juveniles, including parasitic second-stage juveniles (5 dpi) and parasitic third- and fourth-stage juveniles (15 dpi). dpi: days post infection. (d-e) Immunolocalisation with the antiMiPDI1 antibody showed that the MiPDI1 protein was present in the subventral oesophageal glands (SvG). (d) Overlay of images of the differential interference contrast and Alexa Fluor 488 fluorescence. (e) Alexa Fluor 488 fluorescence image. Bar: $20 \mu \mathrm{m}$. (f) Localisation of the 
secreted MiPDI1 protein in the nematode anterior part (black arrow) and at the tip of the stylet (red arrow). (g) Localisation of the secreted MiPDI1 protein in the tip of the stylet and in plant tissue (red arrow). (h-j) MiPDI1 protein accumulated in the nematode anterior part (black arrow), the giant or plant cell wall (red arrows) and in the giant cell (yellow arrow). Micrographs f-j are overlays of images of the DIC, DAPI-stained nuclei and Alexa Fluor 488 fluorescence images. Individual images are presented in Figure S3. N, nematode; *, giant cell. m, metacorpus; Scale bar, $20 \mu \mathrm{m}$.

Fig. 3 The effect of host-derived RNA interference (RNAi) and ectopic expression of $M$. incognita MiPDI1 in Arabidopsis on RKN infection. (a) MiPDI1 expression in three independent, homozygous pdi-Ri lines, two gfp-RNAi lines and the wild type (WT) were determined 10 dpi by RT-qPCR. The data shown are means \pm SE from three independent biological replicates. (b) Phenotypes of nematodes in different Arabidopsis line roots. Acid fuchsin was used to stain Arabidopsis roots after M. incognita infection 35 days. For each line, at least 10 roots were observed, and the experiment was repeated three times. Bars represent $300 \mu \mathrm{m}$. (c) In vivo RNAi of MiPDI1 in Arabidopsis reduced the level of M. incognita infection. Mean numbers of galls and nematodes (parasitic juveniles at any stage and females) were determined at $35 \mathrm{dpi}$ in various Arabidopsis lines. Data are presented as means $\pm \mathrm{SD}$ $(n \geq 30)$. Similar results were obtained in three independent experiments. One representative experiment is shown. (d) MiPDI1 expression led to a reproducible increase in Arabidopsis susceptibility to $M$. incognita. Total numbers of egg masses in two independent MiPDI1 transgenic lines were counted at 35 dpi. Two independent experiments were conducted for each line, and 30 plants were analysed per line. (a, c, d) Different letters indicate statistically significant difference in two-way ANOVA with Tukey's multiple comparisons test $(P<0.05)$.

Fig. 4 MiPDI1 interacts with Arabidopsis and Solanaceae stress-associated proteins SAP12. (a) Pairwise yeast two-hybrid tests were performed to investigate the interactions between MiPDI1 and cysteine proteinase (S1CYP) or SAP12 proteins from S. lycopersicum (S1SAP12), $N$. 
benthamiana (NbSAP12) and A. thaliana (AtSAP12). Left column, yeast cell growth carrying the baits (in pGBKT7 vector) and preys (in pGADT7) grown on SD/-trp-leu medium indicating successful transformation of the yeast with both plasmids; right column, yeast cell growth on the selective triple dropout medium (SD/-trp-leu-his) following the addition of 3-amino-1,2,4triazole (3AT) indicating protein interaction. Yeast cells containing p53 and SV40 were used as positive control. (b) Subcellular localisation of MiPDI1, GFP-S1CYP and SISAP12 in $N$. benthamiana. MiPDI1-eGFP, eGFP, SICYP-RFP and SISAP12-RFP were transiently expressed in $N$. benthamiana leaves. Signals were detected $48 \mathrm{~h}$ after infiltration. Images were captured by confocal microscopy (Zeiss LSM 880, Germany). Scale bar, $20 \mu \mathrm{m}$. (c) Bimolecular fluorescence complementation (BiFC) visualisation of the interaction between MiPDI1 and S1SAP12. $N$. benthamiana leaves were transformed with YFPn-MiPDI1 and S1SAP12-YFPc or SlCYP-YFPc. Images were obtained $36 \mathrm{~h}$ after co-expression. Signals were observed in the cytoplasm in leaves co-infiltrated with YFPn-MiPDI1 and S1SAP12-YFPc. (b, c) At least ten cells from three leaves of three different plants were observed with similar results. YFP, yellow fluorescent protein. Scale bar, $20 \mu \mathrm{m}$ or $50 \mu \mathrm{m}$. (d) Co-immunoprecipitation (Co-IP) analysis of MiPDI1 interacting with SISAP12. FLAG-MiPDI1 or FLAG-GFP was transiently co-expressed with SISAP12-HA or SICYP-HA in tobacco leaves. Co-IP was performed with anti-FLAG M2 affinity gel resin (Sigma-Aldrich), and the isolated protein was detected by western blotting with an anti-FLAG antibody to detect MiPDI1 or eGFP, and an anti-HA antibody to detect SISAP12 or S1CYP. eGFP, enhanced green fluorescent protein.

Fig. 5 Effect on susceptibility to $M$. incognita of virus-induced gene silencing (VIGS) of NbSAP12s and NbCYP in N. benthamiana, and of the A. thaliana sap12 knockout mutant. (a) Levels of $N b S A P 12 s$ and $N b C Y P$ transcripts in $N$. benthamiana following silencing, as assessed by real-time quantitative PCR (RT-qPCR). Error bars represent the standard errors for 10 biological replicates, and the results of two independent experiments were presented. (b) $N$. benthamiana plants in which $N b S A P 12 s$ was silenced were more susceptible to M. incognita, whereas those in which $N b C Y P$ was silenced were not significantly different from the wild type 
in terms of susceptibility, as indicated by the mean numbers of egg masses on plant roots. Error bars represent the mean $\pm \mathrm{SD}(n \geq 15)$. All experiments were performed twice, and at least 15 plants were analysed per treatment. (c) The sap12 mutant line (SALK_014706) was more susceptible to $M$. incognita, as shown by the mean numbers of egg masses and galls in roots. Error bars represent the mean \pm SD $(n \geq 20)$. Two independent experiments were conducted and yielded similar results, with at least 30 plants analysed per treatment. (a, b, c) Different letters indicate statistically significant difference in two-way ANOVA with Tukey's multiple comparisons test $(P<0.05)$. (d) Levels of expression for stress- and defence-related genes in MiPDI1-expressing lines (MiPDI1-1 and MiPDI1-2), the sap12 mutant line (SALK_014706) and the wild type (WT). The genes considered were AtCSD1 (cytosolic Cu/Zn superoxide dismutase), AtCSD2 (chloroplastic $\mathrm{Cu} / \mathrm{Zn}$ superoxide dismutase), AtCOR47 and AtRAB18 (from the dehydrin protein family), AtADHI (catalysing the reduction of acetaldehyde with NADH as reductant), AtEM6 (stress-induced protein), AtNPR1 and AtPRla (SA-mediated defence response marker gene), AtPDF1.2a (encoding ethylene- and jasmonate-responsive plant defences), AtPR4 (ethylene-responsive pathogenesis-related protein). AtOXA1 (AT5G62050) and AtUBP22 (AT5G10790) were used as internal controls. Expression levels were measured by real-time quantitative PCR (RT-qPCR) and the data shown are means $\pm \mathrm{SD}(n=4)$. Asterisks indicate significant differences for single stress- and defence-related gene among different plant lines by one-way ANOVA with Dunnett's multiple comparisons test $\left({ }^{*} P<0.05,{ }^{*} * P<0.01\right.$, *** $P<0.001)$.

\section{Supporting information}

768 Table S1 Primers used in this study.

769 Table S2 Accession numbers used in this study.

770 Table S3 Yeast-two hybrid clones obtained using MiPDI1-mu or MiPDI1 as bait and

771 corresponding gene expression in galls induced by M. incognita.

772 Fig. S1 MiPDI1 probe used for in situ hybridization (ISH).

773 Fig. S2 Western blot analysis verified specificity of MiPDI1 antibody. 
774 Fig. S3 Localization of MiPDI1 in tomato root gall sections during $M$. incognita parasitism.

775 Fig. S4 Verification of homozygous MiPDI1 ectopic expressing Arabidopsis lines by RT-PCR

776 and western blot.

777 Fig. S5 Structure of AtSAP12, NbSAP12 and S1SAP12 proteins.

778 Fig. S6 MiPDI1 could not interact with SlCYP in planta by using BiFC.

779 Fig. S7 Characteristics of $S A P 12$ and $C Y P$ genes, and VIGS phenotypes.

Fig. S8 Verification of homozygous T-DNA insertion mutants of AtSAP12. 


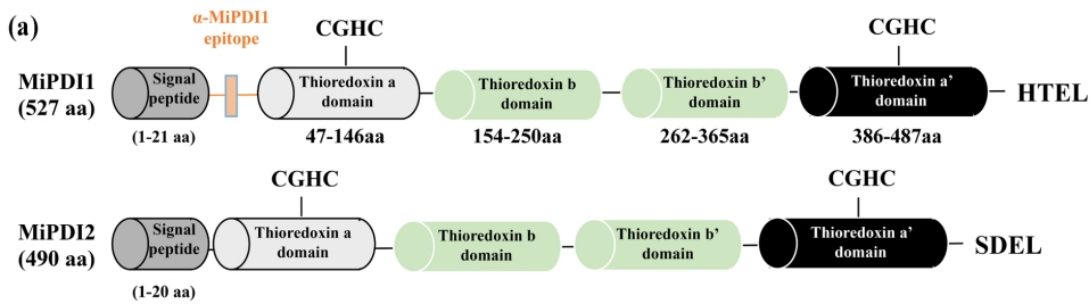

(b)
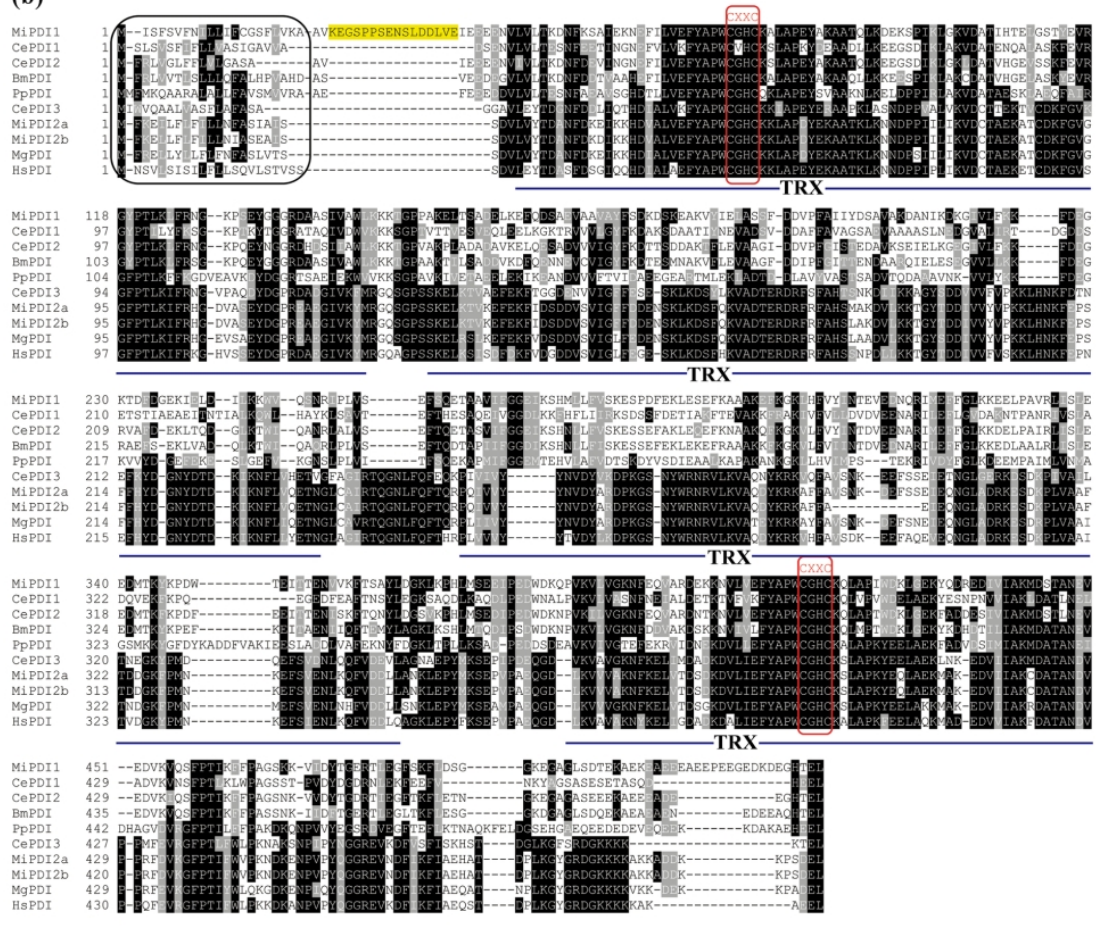

(c)

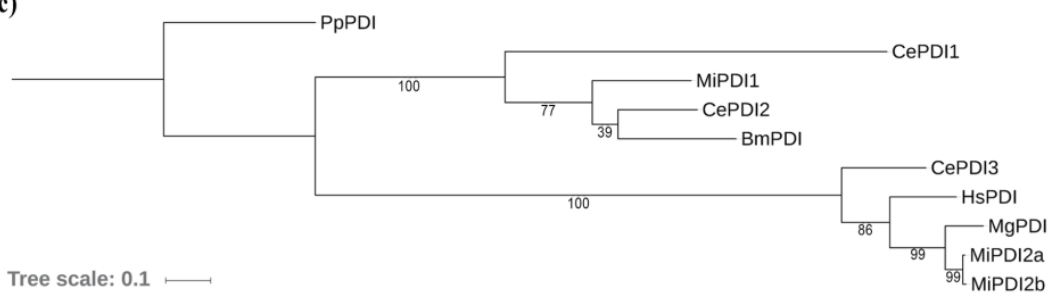

Fig. 1 Primary structure of MiPDIs. (a) Functional domains of MiPDI1 and MiPDI2. MiPDI1 and MiPDI2 have an N-terminal signal peptide for secretion, four thioredoxin domains $\left(a, b, b^{\prime}\right.$, $\left.a^{\prime}\right)$ predicted by an NCBI conserved domain search, and two catalytic domains containing characteristic CGHC active sites in the a and $a^{\prime}$ domains. (b) A ClustalW2 alignment of the PDI-like proteins MiPDI1 and MiPDI2 (from Meloidogyne incognita), CePDI (Caenorhabditis elegans), HsPDI (Heterodera schachtii), MgPDI (Meloidogyne graminicola), BmPDI (Brugia malayi) and PpPDI (Phytophthora parasitica). Identical and highly similar (>75\%) amino-acid residues are highlighted against black background shading, similar (>50\%) amino-acid residues are shown in grey. Yellow background shading indicates the peptide used in the production of the polyclonal anti-MiPDI1 antibody. The sequences for active site CGHC motif are shown in the red frame. The four thioredoxin (TRX) domains are indicated. (c) Maximum likelihood phylogenetic tree of PDI sequences presented in (b). Support for the nodes was calculated with a hundred bootstrap replicates. PpPDI was used as the outgroup. 
Page 33 of 36

Manuscript submitted to New Phytologist for review 


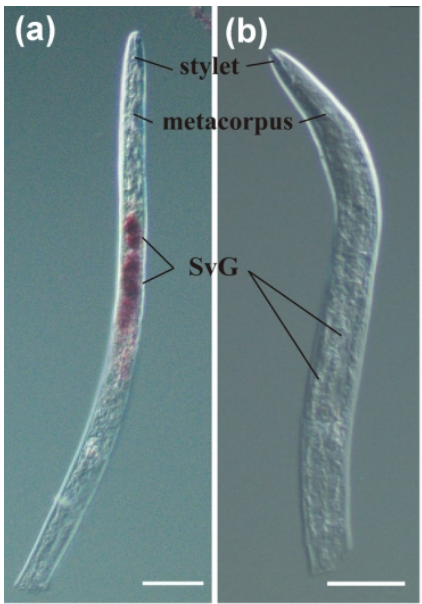

(c)
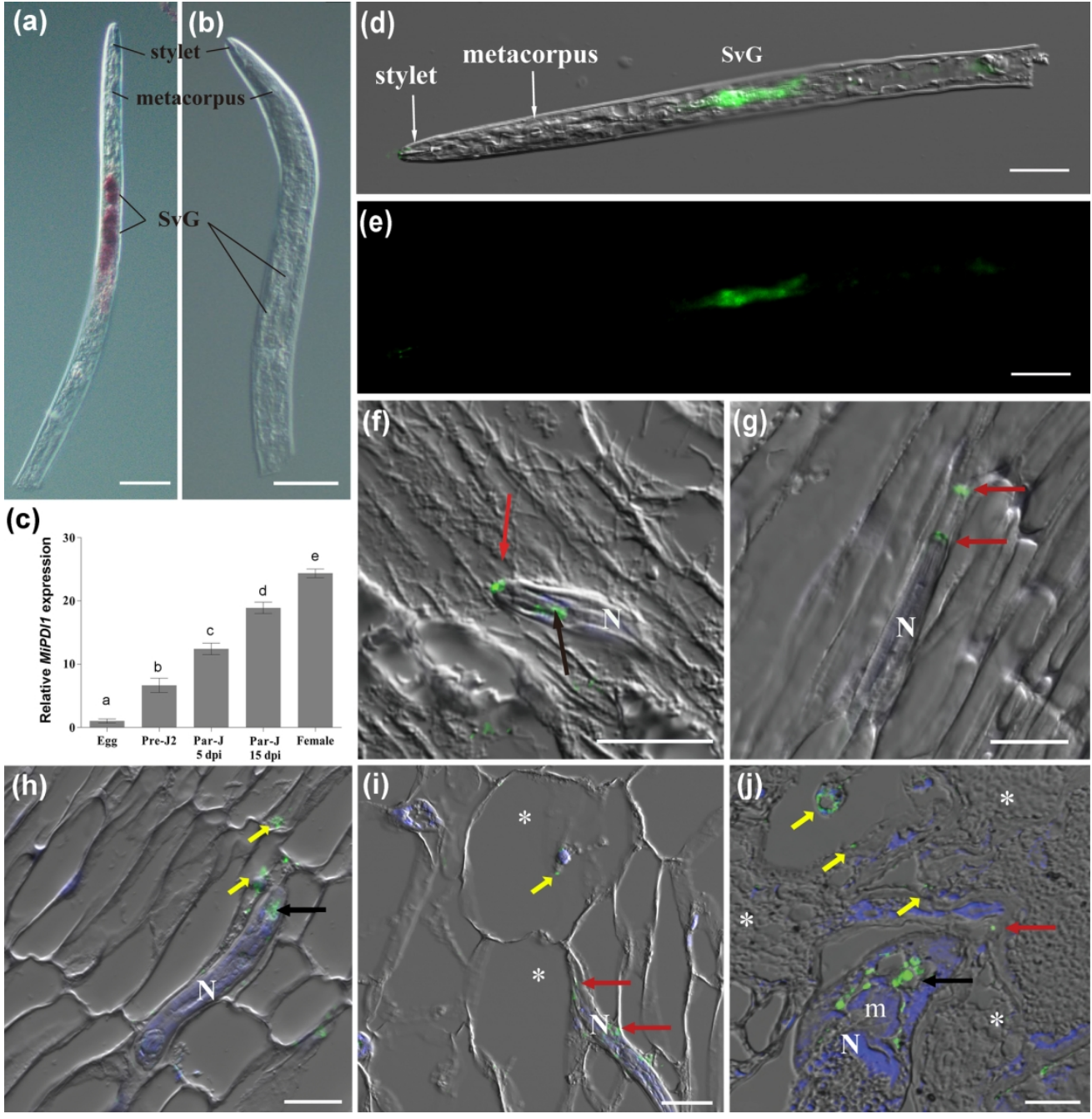

Fig. 2 Localisation and temporal expression pattern of MiPDI. (a) In situ hybridisation (ISH) of digoxigeninlabelled antisense MiPDI1 probe to pre-parasitic M. incognita J2s, showing transcripts expressed in the subventral oesophageal gland (SvG). The stylet and the metacarpus are indicated. (b) ISH with the MiPDI1 sense probe gave no signal. (c) Temporal pattern of MiPDI1 expression. The relative level of MiPDI1 expression throughout nematode development was quantified by RT-qPCR for five different $M$. incognita stages relative to the egg stage (means $\pm \mathrm{SE}$ ). Data were analysed by the 2- $\Delta \Delta \mathrm{Ct}$ method. Different letters indicate statistically significant difference between each column using two-way ANOVA following Tukey's multiple comparisons test $(\mathrm{P}<0.05)$. Pre-J2: preparasitic second-stage juvenile; par-J: parasitic juveniles, including parasitic second-stage juveniles ( $5 \mathrm{dpi}$ ) and parasitic third- and fourth-stage juveniles (15 dpi). dpi: days post infection. (d-e) Immunolocalisation with the anti-MiPDI1 antibody showed that the MiPDI1 protein was present in the subventral oesophageal glands (SvG). (d) Overlay of images of the differential interference contrast and Alexa Fluor 488 fluorescence. (e) Alexa Fluor 488 fluorescence image. Bar: $20 \mu \mathrm{m}$. (f) Localisation of the secreted MiPDI1 protein in the nematode anterior part (black arrow) and at the tip of the stylet (red arrow). (g) Localisation of the secreted MiPDI1 protein in the tip of the stylet and in plant tissue (red arrow). ( $h-j)$ MiPDI1 protein accumulated in the nematode anterior part (black arrow), the giant or plant cell wall (red arrows) and in the giant cell (yellow arrow). Micrographs $f-j$ are overlays of images of the DIC, DAPI-stained nuclei and Alexa Fluor 488 fluorescence images. Individual images are presented in

Figure S3. N, nematode; *, giant cell. m, metacorpus; Scale bar, $20 \mu \mathrm{m}$. 
Page 35 of 36

Manuscript submitted to New Phytologist for review 
(a)

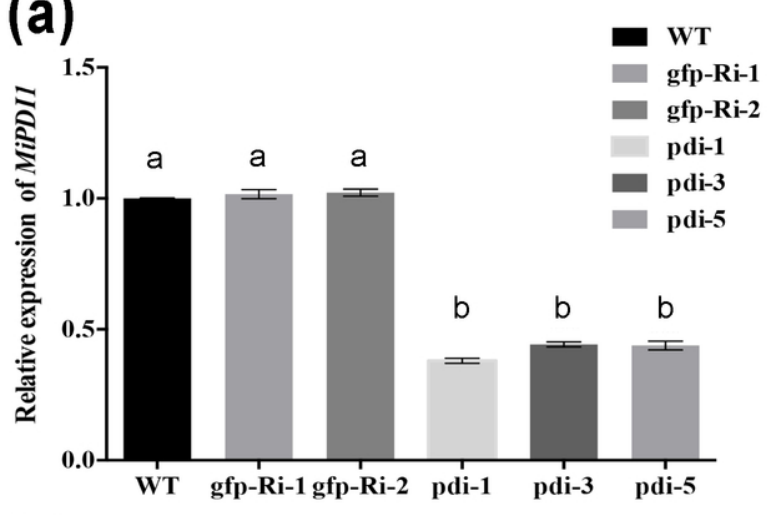

(c)

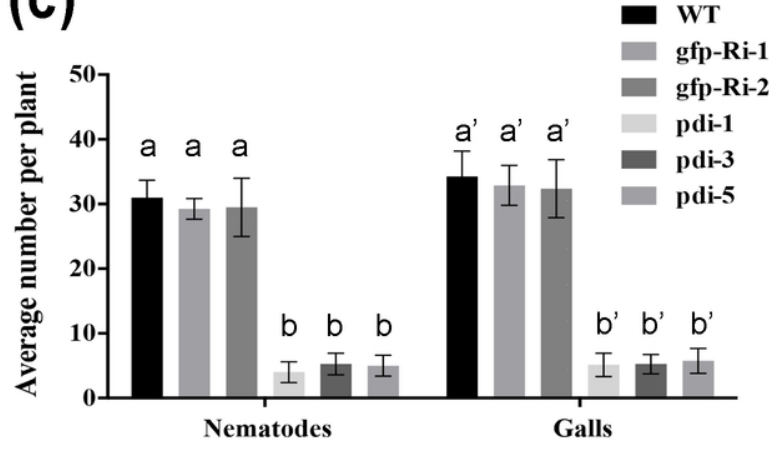

(d)

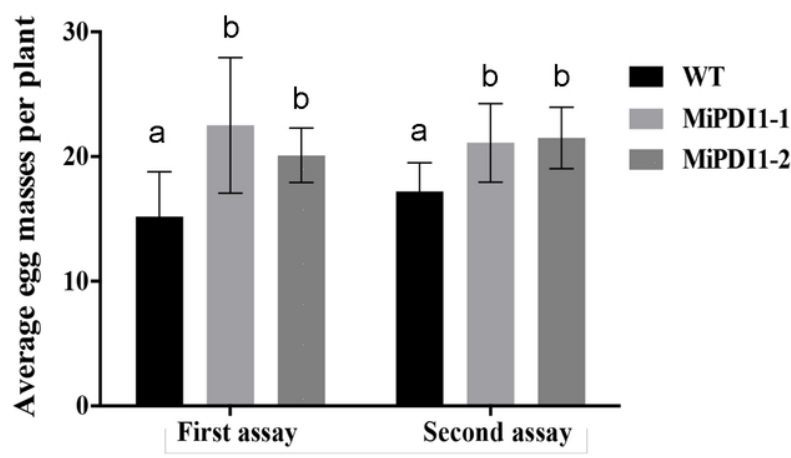

(b)
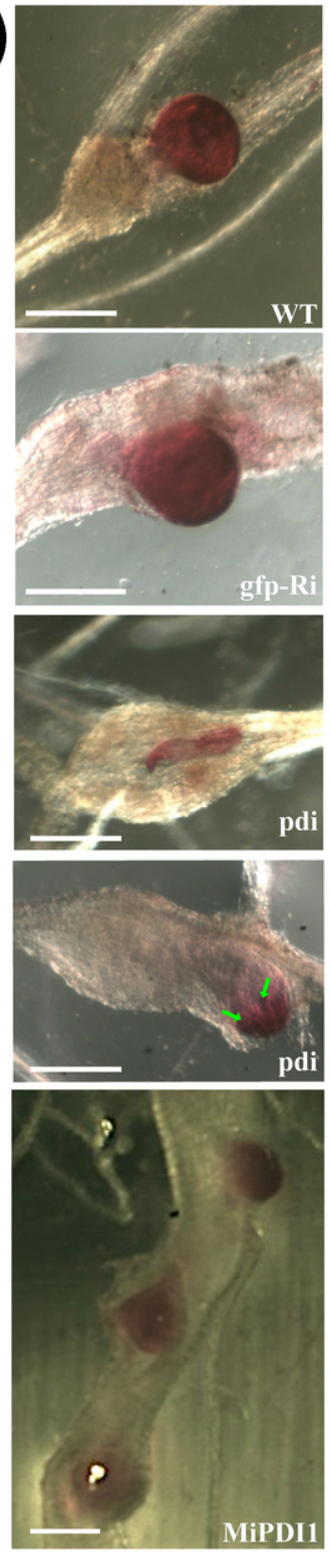

Fig. 3 The effect of host-derived RNA interference (RNAi) and ectopic expression of M. incognita MiPDI1 in Arabidopsis on RKN infection. (a) MiPDI1 expression in three independent, homozygous pdi-Ri lines, two gfp-RNAi lines and the wild type (WT) were determined $10 \mathrm{dpi}$ by RT-qPCR. The data shown are means $\pm \mathrm{SE}$ from three independent biological replicates. (b) Phenotypes of nematodes in different Arabidopsis line roots. Acid fuchsin was used to stain Arabidopsis roots after M. incognita infection 35 days. For each line, at least 10 roots were observed, and the experiment was repeated three times. Bars represent $300 \mu \mathrm{m}$. (c) In vivo RNAi of MiPDI1 in Arabidopsis reduced the level of M. incognita infection. Mean numbers of galls and nematodes (parasitic juveniles at any stage and females) were determined at $35 \mathrm{dpi}$ in various Arabidopsis

lines. Data are presented as means $\pm S D(n \geq 30)$. Similar results were obtained in three independent experiments. One representative experiment is shown. (d) MiPDI1 expression led to a reproducible increase

in Arabidopsis susceptibility to M. incognita. Total numbers of egg masses in two independent MiPDI1 transgenic lines were counted at $35 \mathrm{dpi}$. Two independent experiments were conducted for each line, and 30 plants were analysed per line. ( $a, c, d)$ Different letters indicate statistically significant difference in two-way ANOVA with Tukey's multiple comparisons test $(P<0.05)$. 
Page 37 of 36

$80 \times 106 \mathrm{~mm}(300 \times 300 \mathrm{DPI})$

Manuscript submitted to New Phytologist for review 
(a)
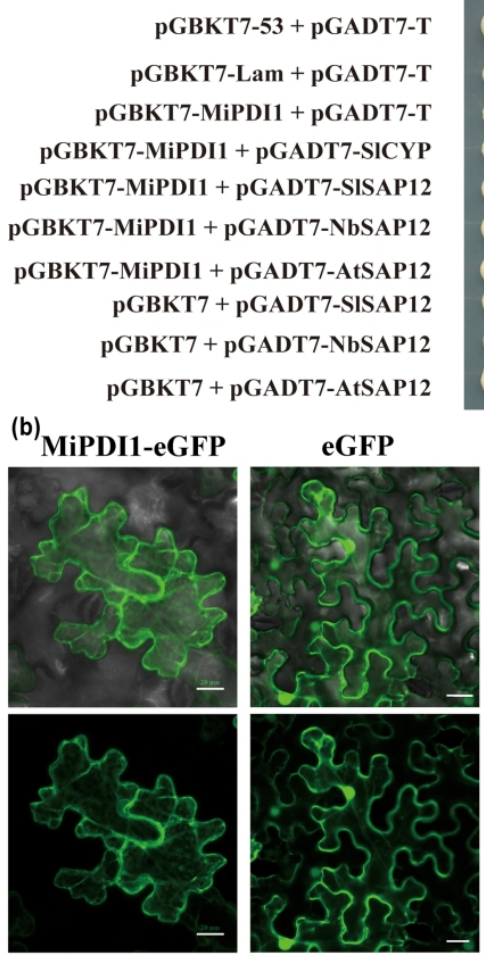

(c)

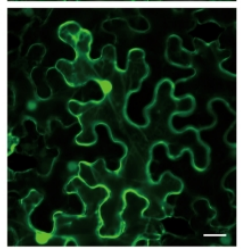

YFPn-MiPDI1+SICYP-YFPc YFPn-MiPDI1+SISAP12-YFPc
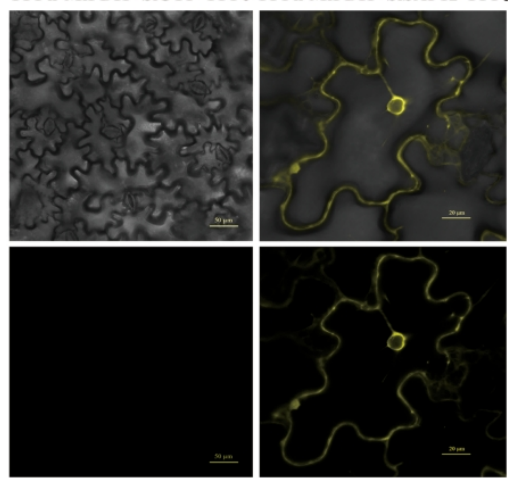

(d)
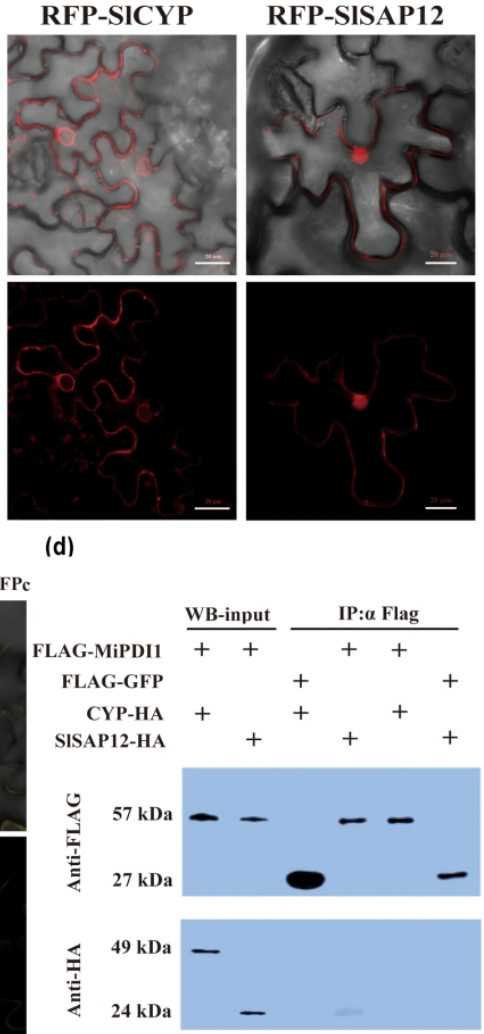

Fig. 4 MiPDI1 interacts with Arabidopsis and Solanaceae stress-associated proteins SAP12. (a) Pairwise yeast two-hybrid tests were performed to investigate the interactions between MiPDI1 and cysteine proteinase (SICYP) or SAP12 proteins from S. Iycopersicum (SISAP12), N. benthamiana (NbSAP12) and A. thaliana (AtSAP12). Left column, yeast cell growth carrying the baits (in pGBKT7 vector) and preys (in pGADT7) grown on SD/-trp-leu medium indicating successful transformation of the yeast with both plasmids; right column, yeast cell growth on the selective triple dropout medium (SD/-trp-leu-his) following the addition of 3-amino-1,2,4-triazole (3AT) indicating protein interaction. Yeast cells containing p53 and SV40 were used as positive control. (b) Subcellular localisation of MiPDI1, GFP-SICYP and SISAP12 in N. benthamiana. MiPDI1-eGFP, eGFP, SICYP-RFP and SISAP12-RFP were transiently expressed in N. benthamiana leaves. Signals were detected $48 \mathrm{~h}$ after infiltration. Images were captured by confocal microscopy (Zeiss LSM 880, Germany). Scale bar, $20 \mu \mathrm{m}$. (c) Bimolecular fluorescence complementation

(BiFC) visualisation of the interaction between MiPDI1 and SISAP12. N. benthamiana leaves were transformed with YFPn-MiPDI1 and SISAP12-YFPC or SICYP-YFPC. Images were obtained $36 \mathrm{~h}$ after coexpression. Signals were observed in the cytoplasm in leaves co-infiltrated with YFPn-MiPDI1 and SISAP12- 
YFPc. (b, c) At least ten cells from three leaves of three different plants were observed with similar results. YFP, yellow fluorescent protein. Scale bar, $20 \mu \mathrm{m}$ or $50 \mu \mathrm{m}$. (d) Co-immunoprecipitation (Co-IP) analysis of MiPDI1 interacting with SISAP12. FLAG-MiPDI1 or FLAG-GFP was transiently co-expressed with SISAP12-HA or SICYP-HA in tobacco leaves. Co-IP was performed with anti-FLAG M2 affinity gel resin (Sigma-Aldrich), and the isolated protein was detected by western blotting with an anti-FLAG antibody to detect MiPDI1 or eGFP, and an anti-HA antibody to detect SISAP12 or SICYP. eGFP, enhanced green fluorescent protein. 
(a)

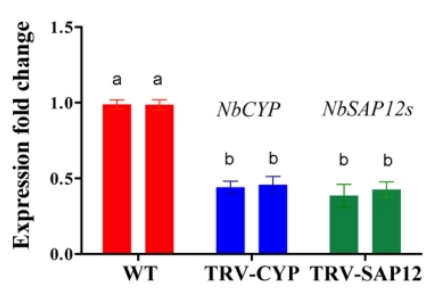

(d) (b)

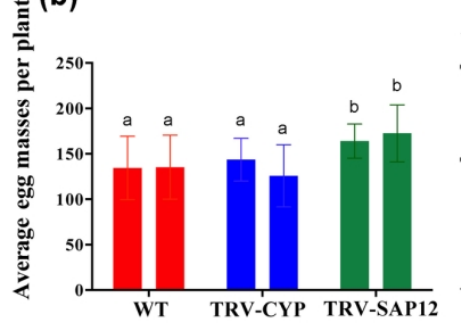

(c)
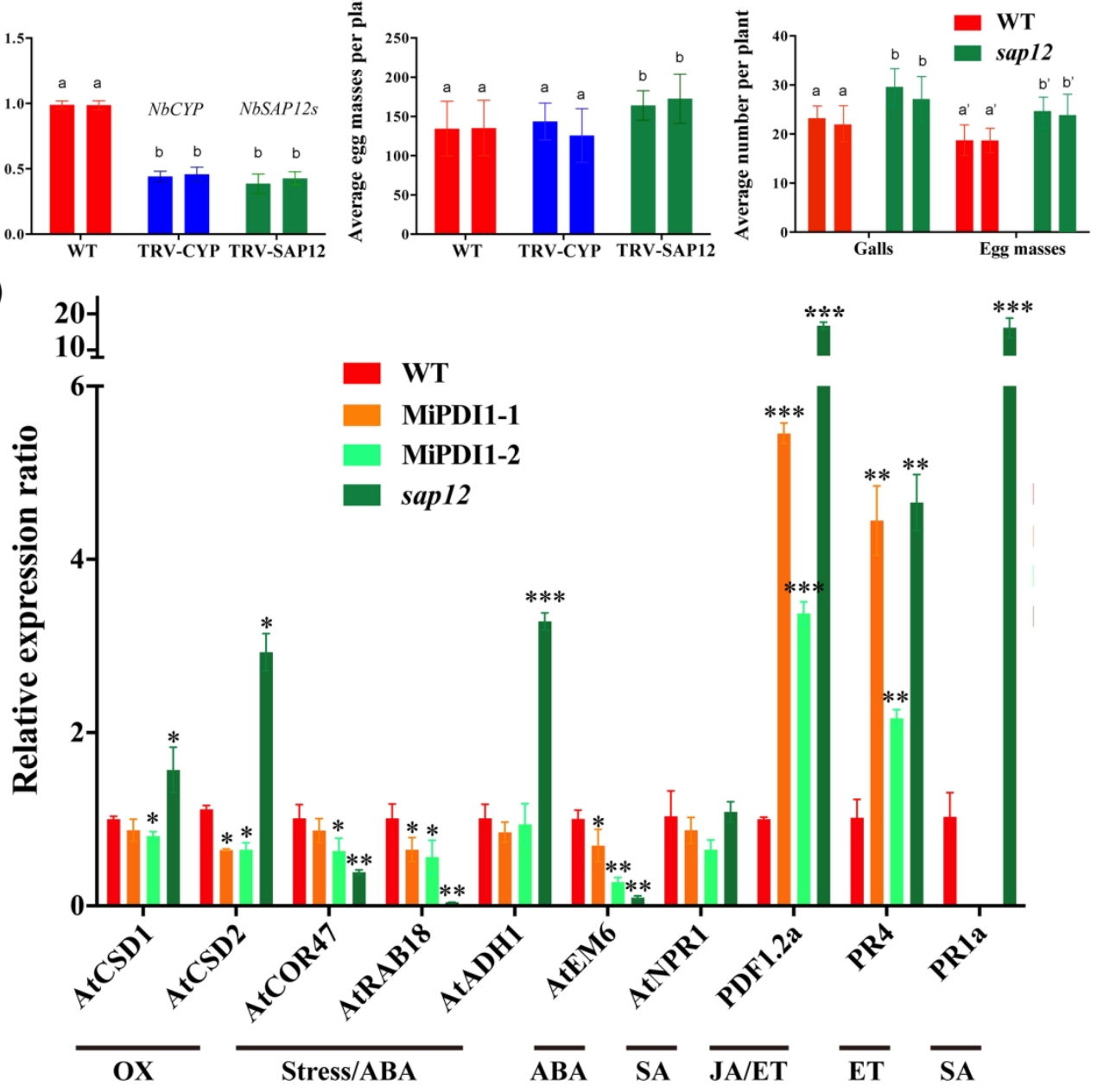

Fig. 5 Effect on susceptibility to M. incognita of virus-induced gene silencing (VIGS) of NbSAP12s and NbCYP in N. benthamiana, and of the A. thaliana sap12 knockout mutant. (a) Levels of NbSAP12s and NbCYP transcripts in N. benthamiana following silencing, as assessed by real-time quantitative PCR (RT-qPCR). Error bars represent the standard errors for 10 biological replicates, and the results of two independent experiments were presented. (b) N. benthamiana plants in which NbSAP12s was silenced were more susceptible to $M$. incognita, whereas those in which NbCYP was silenced were not significantly different from the wild type in terms of susceptibility, as indicated by the mean numbers of egg masses on plant roots. Error bars represent the mean $\pm S D(n \geq 15)$. All experiments were performed twice, and at least 15 plants were analysed per treatment. (c) The sap12 mutant line (SALK_014706) was more susceptible to M. incognita, as shown by the mean numbers of egg masses and galls in roots. Error bars represent the mean \pm SD $(n \geq 20)$. Two independent experiments were conducted and yielded similar results, with at least 30 plants analysed per treatment. (a, b, c) Different letters indicate statistically significant difference in twoway ANOVA with Tukey's multiple comparisons test $(P<0.05)$. (d) Levels of expression for stress- and defence-related genes in MiPDI1-expressing lines (MiPDI1-1 and MiPDI1-2), the sap12 mutant line (SALK_014706) and the wild type (WT). The genes considered were AtCSD1 (cytosolic Cu/Zn superoxide dismutase), AtCSD2 (chloroplastic Cu/Zn superoxide dismutase), AtCOR47 and AtRAB18 (from the dehydrin protein family), AtADH1 (catalysing the reduction of acetaldehyde with NADH as reductant), AtEM6 (stressinduced protein), AtNPR1 and AtPR1a (SA-mediated defence response marker gene), AtPDF1.2a (encoding ethylene- and jasmonate-responsive plant defences), AtPR4 (ethylene-responsive pathogenesis-related protein). AtOXA1 (AT5G62050) and AtUBP22 (AT5G10790) were used as internal controls. Expression levels were measured by real-time quantitative PCR (RT-qPCR) and the data shown are means $\pm S D(n=4)$. 
Asterisks indicate significant differences for single stress- and defence-related gene among different plant lines by one-way ANOVA with Dunnett's multiple comparisons test ( $* \mathrm{P}<0.05, * * \mathrm{P}<0.01, * * * \mathrm{P}<0.001)$.

$160 \times 160 \mathrm{~mm}(300 \times 300 \mathrm{DPI})$ 